\title{
PATHWAY TO THE GALACTIC DISTRIBUTION OF PLANETS: COMBINED SPITZER AND GROUND-BASED MICROLENS PARALLAX MEASUREMENTS OF 21 SINGLE-LENS EVENTS
}

\author{
S. Calchi Novati ${ }^{1,2,3,53}$, A. Gould ${ }^{4,52}$, A. Udalski ${ }^{5,46}$, J. W. Menzies ${ }^{6,47}$, I. A. Bond ${ }^{7,48}$, Y. Shvartzvald ${ }^{8,49}$, \\ R. A. Street ${ }^{9,50,51}$, M. Hundertmark ${ }^{10,11,50,51}$, C. A. Beichman ${ }^{1}$, J. C. Yee ${ }^{12,52,54}$, S. Carey ${ }^{13}$
}

R. Poleski ${ }^{5,4}$, J. Skowron ${ }^{5}$, S. KozŁowski ${ }^{5}$, P. Mróz ${ }^{5}$, P. Pietrukowicz ${ }^{5}$, G. Pietrzyński ${ }^{5,14}$, M. K. Szymański ${ }^{5}$, I. Soszyński ${ }^{5}$, K. ULACZYK ${ }^{5}$, Ł. WYRZYKOWSKI ${ }^{5,15}$

(The OGLE COLlaboration),

M. Albrow ${ }^{16}$, J. P. Beaulieu ${ }^{17,18}$, J. A. R. Caldwell ${ }^{19}$, A. Cassan ${ }^{17}$, C. Coutures ${ }^{17}$, C. Danielski ${ }^{20}$, D. Dominis Prester $^{21}$,

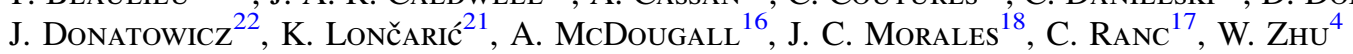

(The PLANET COllaboration),

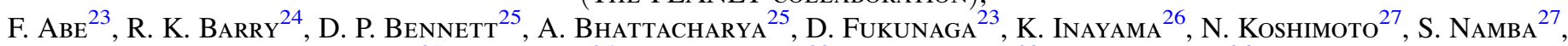
T. Sumi ${ }^{27}$, D. SuZUKi ${ }^{25}$, P. J. Tristram ${ }^{28}$, Y. WakiYama ${ }^{23}$, A. Yonehara ${ }^{26}$

(The MOA COLlaboration),

\section{MAOZ ${ }^{8}$, S. KASPI ${ }^{8}$, M. FRIEDMANN ${ }^{8}$}

(THE Wise GROUP),

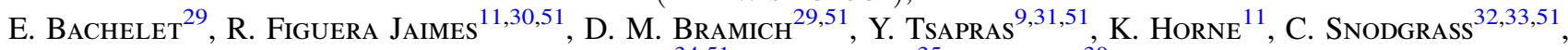
J. Wambsganss ${ }^{34,51}$, I. A. Steele ${ }^{35}$, N. Kains ${ }^{30}$

(The RoboNet COllaboration),

V. Bozza ${ }^{2,36}$, M. Dominik ${ }^{11,50,55}$, U. G. Jørgensen ${ }^{10}$, K. A. Alsubai ${ }^{29}$, S. Ciceri $^{37}$, G. D’ Ago ${ }^{2,36,50}$, T. Haugbølle ${ }^{10}$,

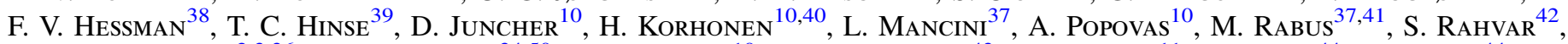
G. Scarpetta ${ }^{2,3,36}$, R. W. Schmidt ${ }^{34,50}$, J. Skottfelt ${ }^{10}$, J. Southworth ${ }^{43}$, D. Starkey ${ }^{11}$, J. Surdej ${ }^{44}$, O. Wertz ${ }^{44}$, M. ZARUCKI ${ }^{3}$

(THE MiNDSTEP CONSORTIUM),

AND

B. S. GAUdi ${ }^{4}$, R. W. PogGe ${ }^{4}$, D. L. DePoy ${ }^{45}$

(THE $\mu$ FUN COLLABORATION)

${ }^{1}$ NASA Exoplanet Science Institute, MS 100-22, California Institute of Technology, Pasadena, CA 91125, USA

${ }^{2}$ Dipartimento di Fisica "E. R. Caianiello," Università di Salerno, Via Giovanni Paolo II, I-84084 Fisciano (SA), Italy

${ }^{3}$ Istituto Internazionale per gli Alti Studi Scientifici (IIASS), Via G. Pellegrino 19, I-84019 Vietri Sul Mare (SA), Italy

${ }^{4}$ Department of Astronomy, Ohio State University, 140 W. 18th Ave., Columbus, OH 43210, USA

${ }^{5}$ Warsaw University Observatory, Al. Ujazdowskie 4, 00-478 Warszawa, Poland

${ }^{6}$ South African Astronomical Observatory, P.O. Box 9, Observatory 7935, South Africa

${ }^{7}$ Institute of Natural and Mathematical Sciences, Massey University, Private Bag 102-904, North Shore Mail Centre, Auckland, New Zealand

${ }^{8}$ School of Physics and Astronomy, Tel-Aviv University, Tel-Aviv 69978, Israel
${ }^{9}$ Las Cumbres Observatory Global Telescope Network, 6740 Cortona Drive, Suite 102, Goleta, CA 93117, USA

${ }^{10}$ Niels Bohr Institutet, Københavns Universitet, Juliane Maries Vej 30, DK-2100 København Ø, Denmark

${ }^{11}$ SUPA, School of Physics \& Astronomy, North Haugh, University of St Andrews, KY16 9SS, Scotland, UK

${ }_{12}^{12}$ Harvard-Smithsonian Center for Astrophysics, 60 Garden St., Cambridge, MA 02138, USA

${ }_{14}^{13}$ Spitzer Science Center, MS 220-6, California Institute of Technology, Pasadena, CA, USA

${ }_{15}^{14}$ Universidad de Concepción, Departamento de Astronomia, Casilla 160-C, Concepción, Chile

${ }^{15}$ Institute of Astronomy, University of Cambridge, Madingley Road, Cambridge CB3 OHA, UK

${ }^{16}$ University of Canterbury, Dept. of Physics and Astronomy, Private Bag 4800, 8020 Christchurch, New Zealand

${ }^{17}$ Institut d'Astrophysique de Paris, UPMC-CNRS, UMR 7095, 98bis Boulevard Arago, F-75014 Paris, France

${ }^{18}$ LESIA, Observatoire de Paris, Section de Meudon 5, place Jules Janssen, F-92195 Meudon, France

${ }^{19}$ McDonald Observatory, 16120 St Hwy Spur 78 \#2, Fort Davis, TX 79734, USA

${ }^{20}$ Institut d'Astrophysique Spatiale, UMR 8617, Université Paris Sud, F-91405 Orsay, France

${ }^{21}$ Department of Physics, University of Rijeka, Radmile Matej vcić 2, 51000 Rijeka, Croatia

22 Technical University of Vienna, Department of Computing,Wiedner Hauptstrasse 10, A-1040 Wien, Austria

${ }^{23}$ Solar-Terrestrial Environment Laboratory, Nagoya University, Nagoya 464-8601, Japan

${ }^{24}$ Astrophysics Science Division, NASA Goddard Space Flight Center, Greenbelt, MD 20771, USA

${ }^{25}$ Department of Physics, University of Notre Dame, Notre Dame, IN 46556, USA

${ }^{26}$ Department of Physics, Faculty of Science, Kyoto Sangyo University, 603-8555 Kyoto, Japan

${ }^{27}$ Department of Earth and Space Science, Graduate School of Science, Osaka University, Toyonaka, Osaka 560-0043, Japan

${ }^{28}$ Mt. John University Observatory, P.O. Box 56, Lake Tekapo 8770, New Zealand

${ }^{29}$ Qatar Environment and Energy Research Institute, Qatar Foundation, P.O. Box 5825, Doha, Qatar

${ }^{30}$ European Southern Observatory, Karl-Schwarzschild-Str. 2, D-85748 Garching bei München, Germany

${ }^{31}$ School of Physics and Astronomy, Queen Mary University of London, Mile End Road, London E1 4NS, UK

${ }^{32}$ The Open University, Walton Hall, Milton Keynes MK7 6AA, UK

${ }^{33}$ Max Planck Institute for Solar System Research, Justus-von-Liebig-Weg 3, D-37077 Göttingen, Germany

${ }^{34}$ Astronomisches Rechen-Institut, Zentrum für Astronomie der Universität Heidelberg (ZAH), Mönchhofstraße 12-14, D-69120 Heidelberg, Germany

${ }_{35}$ Astrophysics Research Institute, Liverpool John Moores University, Liverpool CH41 1LD, UK

${ }^{36}$ Istituto Nazionale di Fisica Nucleare, Sezione di Napoli, Via Cintia, I-80126 Napoli, Italy

${ }^{37}$ Max Planck Institute for Astronomy, Königstuhl 17, D-69117 Heidelberg, Germany

${ }^{38}$ Institut für Astrophysik, Georg-August-Universität Göttingen, Friedrich-Hund-Platz 1, D-37077 Göttingen, Germany 


$$
\begin{aligned}
& { }^{39} \text { Korea Astronomy and Space Science Institute, } 776 \text { Daedeokdae-ro, Yuseong-gu, 305-348 Daejeon, Korea } \\
& { }^{40} \text { Finnish Centre for Astronomy with ESO (FINCA), University of Turku, Väisäläntie 20, FI-21500 Piikkiö, Finland } \\
& { }^{41} \text { Instituto de Astrofísica, Facultad de Física, Pontificia Universidad Católica de Chile, Av.Vicuña Mackenna 4860, 7820436 Macul, Santiago, Chile } \\
& 42 \text { Department of Physics, Sharif University of Technology, P.O. Box 11155-9161 Tehran, Iran } \\
& { }_{43} \text { Astrophysics Group, Keele University, Staffordshire, ST5 5BG, UK } \\
& { }^{44} \text { Institut d'Astrophysique et de Géophysique, Université de Liège, 4000 Liège, Belgium } \\
& \text { Department of Physics and Astronomy, Texas A\&M University, College Station, TX 77843-4242, USA } \\
& \text { Received 2014 November 26; accepted 2015 February 23; published 2015 April 24 }
\end{aligned}
$$

\section{ABSTRACT}

We present microlens parallax measurements for 21 (apparently) isolated lenses observed toward the Galactic bulge that were imaged simultaneously from Earth and Spitzer, which was $\sim 1$ AU west of Earth in projection. We combine these measurements with a kinematic model of the Galaxy to derive distance estimates for each lens, with error bars that are small compared to the Sun's galactocentric distance. The ensemble therefore yields a welldefined cumulative distribution of lens distances. In principle, it is possible to compare this distribution against a set of planets detected in the same experiment in order to measure the Galactic distribution of planets. Since these Spitzer observations yielded only one planet, this is not yet possible in practice. However, it will become possible as larger samples are accumulated.

Key words: gravitational lensing: micro - planetary systems - planets and satellites: dynamical evolution and stability

\section{INTRODUCTION}

It has been known for $50 \mathrm{yr}$ (Liebes 1964; Refsdal 1964) that microlensing measurements are plagued by a severe degeneracy between the lens mass $M$, the source-lens relative parallax $\pi_{\text {rel }}=\mathrm{AU}\left(D_{L}^{-1}-D_{S}^{-1}\right)$, and the geocentric lens-source relative proper motion $\boldsymbol{\mu}_{\text {geo }}$ (Gaudi 2012, Equations (1) and (17)),

$$
\begin{gathered}
t_{\mathrm{E}}=\frac{\theta_{\mathrm{E}}}{\mu_{\mathrm{geo}}} ; \quad \theta_{\mathrm{E}}^{2} \equiv \kappa M \pi_{\mathrm{rel}} ; \\
\kappa \equiv \frac{4 G}{c^{2} \mathrm{AU}} \simeq 8.14 \frac{\mathrm{mas}}{M_{\odot}} .
\end{gathered}
$$

Here $\theta_{\mathrm{E}}$ is the angular Einstein radius and $t_{\mathrm{E}}$ is the Einsteinradius crossing time in the ground-based reference frame. It has also been known for $50 \mathrm{yr}$ (Refsdal 1966) that the best way to systematically ameliorate this degeneracy is to observe the events simultaneously from solar orbit in order to measure the microlens parallax vector $\pi_{\mathrm{E}}$,

$$
\pi_{\mathrm{E}} \equiv \frac{\pi_{\mathrm{rel}}}{\theta_{\mathrm{E}}} \frac{\boldsymbol{\mu}}{\mu},
$$

where $\boldsymbol{\mu}$ can be the lens-source relative proper motion in either the geocentric or heliocentric frame, in which cases $\pi_{\mathrm{E}}$ is the representation in the same frame. (Note that, as the ratio of two angles, $\pi_{\mathrm{E}}$ is dimensionless.) If $\pi_{\mathrm{E}}$ is measured, one obtains

\footnotetext{
46 The OGLE collaboration.

47 The PLANET collaboration.

48 The MOA collaboration.

49 The Wise group.

50 The RoboNet collaboration.

${ }^{51}$ The MiNDSTEp consortium.

52 The $\mu$ FUN collaboration.

53 Sagan Visiting Fellow.

54 Sagan Fellow.

55 Royal Society University Research Fellow.
}

strong constraints on $M$ and $\pi_{\text {rel }}$ from

$$
M=\frac{\theta_{\mathrm{E}}}{\kappa \pi_{\mathrm{E}}}=\frac{\mu_{\mathrm{geo}} t_{\mathrm{E}}}{\kappa \pi_{\mathrm{E}}}, \quad \pi_{\mathrm{rel}}=\theta_{\mathrm{E}} \pi_{\mathrm{E}}=\mu_{\mathrm{geo}} t_{\mathrm{E}} \pi_{\mathrm{E}} .
$$

Hence, even if $\theta_{\mathrm{E}}$ is not measured (as it almost never is for single-lens microlenses), $M$ and $\pi_{\text {rel }}$ can be estimated fairly robustly just from the fact that the great majority of the

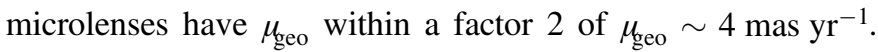
However, without the additional information from $\pi_{\mathrm{E}}$, the three physical quantities $M, \pi_{\text {rel }}$, and $\mu_{\text {geo }}$ cannot be disentangled from the single measured parameter $t_{\mathrm{E}}$, so that, for example, $M$ remains uncertain by an order of magnitude (Gould 2000).

Nevertheless, before 2014, there was only one space-based parallax measurement out of more than 10,000 recorded microlensing events: Dong et al. (2007) used Spitzer to measure the microlens parallax of a rare (almost unique) bright event toward the Small Magellanic Cloud, OGLE-2005-SMC-001.

Spitzer (Werner et al. 2004) has several advantages but also important disadvantages as a possible "microlens parallax satellite" for observations toward the Galactic bulge. First, of course, it is in solar orbit, gradually drifting behind Earth at somewhat more than $0.1 \mathrm{AU} \mathrm{yr}^{-1}$. Hence, by now it trails Earth by almost $90^{\circ}$. Second, at $3.6 \mu \mathrm{m}$, its IRAC camera (Fazio et al. 2004) has relatively good resolution of $\sim 2^{\prime \prime}$, not much worse than the resolutions of the ground-based surveys that discover and monitor the events. Third, it can be pointed at targets on relatively short notice. This is important because microlensing events typically peak (and then decline) within a few weeks of their discovery. Hence, either the satellite must be able to respond quickly (Refsdal 1966; Gould 1994) or it must, like ground observatories, survey an extended field in hope of detecting events from previously unidentified sources (Gould \& Horne 2013).

Spitzer's most important disadvantage is that due to Sunangle viewing restrictions, it can observe any given target that lies near the ecliptic (including the entire Galactic bulge, which hosts $>99 \%$ of all recorded microlensing events) for only two $\sim 38$-day intervals per year. Moreover, during only one of these is it possible to simultaneously observe the bulge from Earth (and so measure parallaxes). Second, while Spitzer's real-time 
response can in principle be extremely rapid, such rapid responses are also very disruptive to its overall mission and science return. By contrast, the normal (non-disruptive) response time to unexpected requests for data is of order a month, which would be useless for the great majority of microlensing events. For completeness, we mention that the fact that Spitzer observes at wavelengths far redward of those used for ground-based microlensing observations was initially believed to be a problem (e.g., Gould 1999), but this now appears to be relatively minor (e.g., Yee et al. 2015).

The observations reported here derive from a $100 \mathrm{hr}$ "pilot program" awarded by the Director to demonstrate the feasibility of Spitzer microlens parallax observations. The scientific objectives were of course driven by the overall potential of Spitzer to determine microlens masses, particularly for planetary events. However, these objectives were also sculpted by the challenges discussed in the previous paragraph, and in particular by the need to demonstrate concretely that these challenges could be met. For example, working with Spitzer operations, we developed a new observing protocol for "regular" (non-disruptive) target-of-opportunity observations with a turnaround of 3-9 days. The times of the microlensing observations were preplanned to occur in blocks approximately once per $24 \mathrm{hr}$, but the targets for a given week were not finalized until a few days before they were uploaded to the spacecraft (Section 2 and Figure 1 of Udalski et al. 2015 and also Section 2.2 of this paper). In particular, the weekly observing decisions made under this protocol were aimed primarily at maximizing the number of successful parallax measurements, while making an extra effort to measure parallaxes for as many binary and planetary events as possible.

An alternative strategy might have been to develop purely objective criteria for the weekly choices of targets and cadences. The ensemble of parallax measurements of isolated lenses made using such an objective protocol could then be forward modeled to extract the underlying mass function, as envisaged by Han \& Gould (1995).

The reasons for not using purely objective criteria were threefold. First, as stated above, the overwhelming objective was to determine feasibility, which can best be done by learning from successful measurements. Second, it is very difficult to develop objective criteria without concrete experience (exactly the point of a "pilot program"). Finally, the lens mass function is not the most important scientific result that can be extracted from an ensemble of isolated-lens measurements.

Rather, the most critical application of an ensemble of isolated-lens parallaxes is to serve as the comparison sample by which the planet detections can be transformed into a measurement of the Galactic distribution of planets. That is, as long as the planetary events are not chosen for Spitzer observations because they are known to have planets, then the planetary events can be considered to be "drawn fairly" from the ensemble of (mainly) isolated-lens events, regardless of whether the process by which the latter are chosen can be modeled or not. This also means that if, during successive years of observations, the selection criteria change, the planet sample and the isolated-lens sample can each be concatenated, and they will still yield a fair comparison. This situation is analogous to the selection of high-magnification events for intensive follow-up that led to the first microlens-planet frequency analysis (Gould et al. 2010), the most relevant point in both cases being that events are selected for observations without regard to whether or not they have planets in them.

Now, since there was only one planet ${ }^{56}$ in the Spitzer "pilot program" sample (Udalski et al. 2015), it is not yet possible to derive a Galactic distribution of planets. Nevertheless, it is important to make an initial effort to measure the distance distribution of the isolated lens sample, partly to learn practically how to do this from real data and partly to understand what type of lenses were effectively selected by the selection procedures used in the "pilot program." Even though these procedures cannot be comprehensibly quantified, they do have quantifiable elements (like delay times of 3-9 days) that by themselves select for certain types of lenses. Even a qualitative understanding of these effects may influence the choice of objective selection criteria in future years. Thus, although it is clearly too early to measure the Galactic distribution of planets, it is actually quite urgent to begin those components of the analysis that can be done.

Making a statistical estimate of the distance distribution of the ensemble of isolated lenses requires that a probability distribution be assigned to the distance of each lens. In general, this probability distribution will be much more compact if the well-known parallax degeneracy (Refsdal 1966; Gould 1994) is broken, as it was by Yee et al. (2015) for the case of OGLE2014-BLG-0939. That is, because $u$ (and so $u_{0}$ ) enters the lensing magnification equation quadratically (Equation (8) below), space-based parallax measurements generically have a fourfold degeneracy in the vector microlens parallax $\pi_{\mathrm{E}}$,

$$
\pi_{\mathrm{E}}=\frac{\mathrm{AU}}{D_{\perp}}\left(\frac{\Delta t_{0}}{t_{\mathrm{E}}}, \Delta u_{0, \pm, \pm}\right),
$$

where the $x$-axis is defined by the direction of the projected Earth-satellite separation vector $\boldsymbol{D}_{\perp}, \Delta t_{0}=t_{0, \text { sat }}-t_{0, \oplus}$ is the difference in times of maximum as seen at the two locations, $\Delta u_{0,-, \pm}= \pm\left(\left|u_{0, \text { sat }}\right|-\left|u_{0, \oplus}\right|\right)$ is the difference in impact parameters assuming that they are on the same side of the lens, and $\Delta u_{0,+, \pm}= \pm\left(\left|u_{0, \text { sat }}\right|+\left|u_{0, \oplus}\right|\right)$ is their difference assuming that they are on the opposite sides. While the two solutions $\Delta u_{0,-, \pm}$ (or two solutions $\Delta u_{0,+, \pm}$ ) yield very similar $\pi_{\mathrm{E}}$ (so $M$ and $\pi_{\text {rel }}$ ), these two sets of solutions can have very different $\pi_{\mathrm{E}}$ from each other. The overall sign of $\Delta u_{0}$, which is designated by the second " \pm " subscript, is positive if $u_{0, \oplus}>0$, which by convention occurs if "the lens passes the moving source on its right as seen from Earth" (Gould 2004). See his Figure 2.

\footnotetext{
56 In fact, OGLE-2014-BLG-0298, which showed a perturbation that was strongly suspected to be planetary in nature well before the commencement of Spitzer observations, was aggressively monitored during this campaign. However, exactly because these observations were triggered by the (suspected) presence of a planet, this event is not part of the "fair sample" and is therefore not considered in the present work. The value of these Spitzer observations, as with Spitzer observations of known binary microlensing events, is to measure the mass of a potentially interesting object, rather than for statistical studies. To date, planetary anomalies far out on the rising wing of the light curve (like OGLE-2014-BLG-0298) have constituted roughly $8 \%$ of all planetary events, so elimination of these events from the Galactic distribution sample is not likely to be a major loss. However, if future planet surveys have more uniform light-curve coverage than past ones (e.g., Henderson et al. 2014), then this fraction will increase somewhat.
} 


\subsection{Rich Argument}

The present ensemble of parallax measurements provides the first opportunity to test an idea that to our knowledge was first suggested by J. Rich (ca. 1997, private communication), but never (to our knowledge) written up. Rich's original idea was that the two components of $\pi_{\mathrm{E}} D_{\perp} / \mathrm{AU}$ (namely, $\Delta \tau=\Delta t_{0} / t_{\mathrm{E}}$ and $\left.\Delta u_{0}\right)$ should in general be of the same order. This is true for different classes of lenses for different reasons. If the lens is in the bulge, then the direction of relative proper motion $\boldsymbol{\mu}$ (and so $\pi_{\mathrm{E}}$ ) is nearly randomly distributed over a circle. Similarly, if the lens is close to the Sun (i.e., within about $1 \mathrm{kpc}$ ), then the direction of proper motion is primarily determined by the lens peculiar motion and is again basically random. Finally, if the lens is at intermediate distances in the Galactic disk, then its proper motion should be roughly aligned with Galactic rotation, which in ecliptic coordinates (relevant since $\boldsymbol{D}_{\perp}$ is closely aligned with the ecliptic) has comparable components.

According to Rich's original idea, in the case that the true solution is one of $\Delta u_{0,-, \pm}$ solutions, the two components will generally be roughly equal $|\Delta \tau| \sim\left|\Delta u_{0,-, \pm}\right|$. If these components are themselves small, $\left|\Delta u_{0,-, \pm}\right| \ll\left|u_{0, \oplus}\right|$, then the components for the other solution will be highly unequal, $\left|\Delta u_{0,+, \pm}\right| \sim 2\left|u_{0, \oplus}\right| \gg\left|\Delta u_{0,-, \pm}\right|$ and consequently $\left|\Delta u_{0,+, \pm}\right| \gg|\Delta \tau|$. Hence, seeing such roughly equal components for one solution and highly unequal components for the other, one should conclude that the first solution is probably correct.

In the course of working on the events in this paper, we realized that Rich's argument can be extended to apply constraints from two degrees of freedom, rather than just one. This increases the argument's statistical power considerably. Properly speaking, it should then be called the "Extended Rich argument," but for simplicity we continue to simply say "Rich argument." We begin by noting that the parallax amplitude basically has a twofold degeneracy, which we denote $\pi_{\mathrm{E}, \pm}$, corresponding to ${ }^{57}\left(\left|\Delta u_{0, \pm}\right|, \Delta \tau\right)$. One of these is the actual parallax $\pi_{\mathrm{E} \text {,true }}$, and the other is spurious, $\pi_{\mathrm{E} \text {,false }}$. However, it is often the case that the light curve does not distinguish between these. Nevertheless, we can define a theoretical quantity

$$
\begin{aligned}
\epsilon & =\frac{\pi_{\mathrm{E}, \text { false }}}{\pi_{\mathrm{E}, \text { true }}}=\left(\frac{\left(\left|u_{0, \mathrm{sat}}\right|-\left|u_{0, \oplus}\right|\right)^{2}+(\Delta \tau)^{2}}{\left(\left|u_{0, \mathrm{sat}}\right|+\left|u_{0, \oplus}\right|\right)^{2}+(\Delta \tau)^{2}}\right)^{ \pm 1 / 2} \\
& =\left(\frac{\left(\left|\Delta u_{0,-}\right|\right)^{2}+(\Delta \tau)^{2}}{\left(\left|\Delta u_{0,+}\right|\right)^{2}+(\Delta \tau)^{2}}\right)^{ \pm 1 / 2}
\end{aligned}
$$

where the sign refers to the cases $\pi_{\mathrm{E} \text {,true }}=\pi_{\mathrm{E}, \pm}$. Hence, for $\pi_{\mathrm{E}, \text { true }}=\pi_{\mathrm{E},+}, \epsilon<1$, and if $\pi_{\mathrm{E},+} \gg \pi_{\mathrm{E},-}$, then $\epsilon \ll 1$. We can test the hypothesis that $\pi_{\mathrm{E} \text {,true }}=\pi_{\mathrm{E},+} \gg \pi_{\mathrm{E},-}$ by asking what is the probability of $\epsilon \leqslant \epsilon_{0}$ where $\epsilon_{0} \ll 1$. This can be divided into two questions: first, what is the prior probability of $\epsilon<1$ given that $\pi_{\mathrm{E}}$ has some given true value, and second, what is the conditional probability $\epsilon<\epsilon_{0}$ given that $\epsilon<1$.

The first probability (namely, that $\pi_{\mathrm{E} \text {,true }}=\pi_{\mathrm{E},+}$ ) is certainly less than unity, and typically of order one-half. We do not further investigate this probability because it depends on the

\footnotetext{
$\overline{57}$ For simplicity of notation we will neglect the second \pm in $\Delta u_{0, \pm, \pm}$ for the remainder of this section.
}

details of event selection and because its specific value has marginal impact on the overall result.

If $\epsilon \ll 1$, then $\left|\Delta u_{0,-}\right| \lesssim \epsilon\left|\Delta u_{0,+}\right|$ and $\Delta \tau \lesssim \epsilon\left|\Delta u_{0,+}\right|$. Under this hypothesis, the latter condition gives highly unequal components for $\pi_{\mathrm{E} \text {,true }}=\pi_{\mathrm{E},+}$, implying a very special angle $\alpha$ for the lens-source relative motion with respect to the direction perpendicular to the Earth-satellite axis, $|\sin \alpha|<\epsilon$, whereas a priori, $\alpha$ could assume any direction over the circle. This is the basis of the original Rich argument. However, the first condition also constrains $\left|\Delta u_{0,-}\right|$ to a very narrow interval relative to the full range of possibilities $-\left|\Delta u_{0,+}\right|<\left|\Delta u_{0,-}\right|<\left|\Delta u_{0,+}\right|$ over which this quantity would be expected to be uniformly distributed. Eliminating duplicate geometries, we should evaluate the probability that $\left[\alpha^{2}+\left(\Delta u_{0,-} / \Delta u_{0,+}\right)^{2}\right]<\epsilon_{0}^{2}$ under conditions where $\alpha$ is uniformly distributed over $[-\pi / 2, \pi / 2]$ and $\left(\Delta u_{0,-} / \Delta u_{0,+}\right)$ is uniformly distributed over $[-1,1]$. This probability is just

$$
P\left(\epsilon<\epsilon_{0} \mid \epsilon<1\right)=\frac{\pi \epsilon_{0}^{2}}{2 \pi}=\frac{\epsilon_{0}^{2}}{2} .
$$

That is, the probability of $\epsilon<\epsilon_{0} \ll 1$ (which requires $\left.\pi_{\mathrm{E} \text {,true }}=\pi_{\mathrm{E},+}\right)$ is very small.

We next note that if $\pi_{\mathrm{E} \text {,true }} \ll 1$, then the probability of $\epsilon \gg 1$ is of order unity. This is because $\pi_{\mathrm{E} \text {,true }} \ll 1$ requires that $\left|\Delta u_{0}\right| \ll 1$ (and $\left.\Delta \tau \ll 1\right)$. Hence, under typical conditions, i.e., $u_{0} \sim \mathcal{O}(0.5)$, we have $\Delta u_{0,+} \sim 2\left|u_{0}\right| \sim \mathcal{O}(1) \gg \Delta u_{0,-}$ and similarly $\Delta u_{0,+} \gg \Delta \tau$.

Hence, if we find $\pi_{\mathrm{E},+} \gg \pi_{\mathrm{E},-}$, then it is highly likely that the $\pi_{\mathrm{E},-}$ solution is correct. This is because, if $\pi_{\mathrm{E},-}$ is correct, then we naturally expect the alternate solution $\left(\pi_{\mathrm{E},+}\right)$ to be much bigger (i.e., $\left|\Delta u_{0,+}\right| \gg\left|\Delta u_{0,-}\right|$ and $\left.\left|\Delta u_{0,+}\right| \gg \Delta \tau\right)$. However, if the $\pi_{\mathrm{E},+}$ solution were correct, then we would expect the alternate solution $\left(\pi_{\mathrm{E},-}\right)$ to be of the same general order, and, in particular, the chance that the alternate solution was as small as observed or smaller would be $\epsilon^{2} / 2$.

Such an argument cannot be considered decisive in any particular case because the proper motion can by chance be very nearly perpendicular to $\boldsymbol{D}_{\perp}$ and the values of $\Delta u_{0}$ as seen from Earth and the satellite can by chance happen to have very nearly equal magnitudes but opposite signs. Nevertheless, if the objective is to find the cumulative distribution of lens distances (rather than to securely determine the distance to a particular lens), then it is appropriate to give unequal-component solutions lower statistical weight when combining the distance estimates of the ensemble to form a cumulative distribution.

\section{OBSERVATIONS}

\subsection{OGLE Observations}

All 21 of the events analyzed in this paper were discovered by the Optical Gravitational Lens Experiment (OGLE) based on observations with the $1.4 \mathrm{deg}^{2}$ camera on its $1.3 \mathrm{~m}$ Warsaw Telescope at the Las Campanas Observatory in Chile using its Early Warning System real-time event detection software (Udalski et al. 1994; Udalski 2003). The observations reported here are entirely in $I$ band, although some $V$ observations were also taken with the aim of determining the source color. The specific role of such source-color measurements in the present study is discussed in Section 2.4. 


\subsection{Spitzer Observations}

The structure of our Spitzer observing protocol is described in detail in Section 3.1 of Udalski et al. (2015). In brief, observations were made during $382.63 \mathrm{hr}$ windows between HJD' $\equiv$ HJD 2,450,000 = 6814.0 and 6850.0. Each observation consisted of six dithered $30 \mathrm{~s}$ exposures in a fixed pattern using the $3.6 \mu \mathrm{m}$ channel on the IRAC camera. Observation sequences were uploaded to Spitzer operations on Mondays at UT 15:00, for observations to be carried out Thursday to Wednesday (with slight variations). As described in Udalski et al. (2015), J.C.Y. and A.G. balanced various criteria to determine which targets to observe and how often. In general, there were too many targets to be able to observe all viable targets during each epoch. The relation between weekly "decision dates" and subsequent observations is illustrated in Figure 1 of Udalski et al. (2015).

With three exceptions, the OGLE alerts for all 21 events occurred prior to the first "decision date" (June 2 UT 15:00, HJD' 6811.1). The alerts for OGLE-2014-BLG-1021, OGLE2014-BLG-1049, and OGLE-2014-BLG-1147 were announced on June 4, 6, and 18, respectively. Hence, the first two could be observed only during 4 weeks, while the third could be observed only during the final 2 weeks.

Table 1 lists the equatorial coordinates, ecliptic latitude, and number of Spitzer observations for each event. The ecliptic latitude is important because in the limit that both the event and the Spitzer spacecraft were directly on the ecliptic, the directional degeneracies $\Delta u_{0,-, \pm}$ and $\Delta u_{0,+, \pm}$ could not be broken, even in principle (Jiang et al. 2005; Skowron et al. 2011).

\subsection{Additional Light-curve Data}

Additional light-curve data were obtained for a total of 15 of the 21 events reported here from a total of 13 telescopes. The MOA collaboration (Bond et al. 2001; Sumi et al. 2013) obtained data on seven events as part of their normal survey operations using a broad R/I filter on their $1.8 \mathrm{~m}$ telescope at Mt. John, New Zealand. Similarly, the Wise Collaboration (Shvartzvald \& Maoz 2012) obtained survey data on five events using an $I$-band filter on their $1.0 \mathrm{~m}$ telescope at Mitzpe Ramon, Israel.

Four other teams specifically targeted the Spitzer sample for follow-up observations, all in $I$ band (or SDSS $i$ band). The PLANET collaboration (Albrow et al. 1998) observed six events using the $1.0 \mathrm{~m}$ Elizabeth telescope at Sutherland, South Africa. The RoboNet/LCOGT (Las Cumbres Observatory Global Telescope Network) collaboration (Tsapras et al. 2009) observed a total of four events from a total of eight $1.0 \mathrm{~m}$ telescopes in CTIO, Chile, Sutherland, South Africa, and Siding Spring, Australia. The MiNDSTEp (Microlensing Network for the Detection of Small Terrestrial Exoplanets) consortium (Dominik et al. 2010) observed four events from their $1.54 \mathrm{~m}$ telescope at ESO La Silla, Chile, and four events using the $0.35 \mathrm{~m}$ Salerno University telescope in Salerno, Italy.

Of the 21 events, $(6,9,1,1,2,1,1)$ were observed by $(1,2$, $3,4,9,10,11)$ telescopes, respectively. We refer to Table 1 for full details on the additional data set used.

\subsection{Additional Color Data}

The $\mu$ FUN (Microlensing Follow Up Network) collaboration obtained a very limited quantity of data on each of the 21 events using the ANDICAM (DePoy et al. 2003) dichroic camera on the SMARTS-CTIO $1.3 \mathrm{~m}$ telescope. These observations were made simultaneously in $I$ and $H$ band and were for the specific purpose of inferring the $I-[3.6]$ source color using an $(I-H)$ versus $(I-[3.6])$ instrumental colorcolor diagram. Yee et al. (2012) demonstrated for the case of MOA-2011-BLG-293 that this color-color method could reliably constrain the source flux even if a given data set lacked sufficient coverage for an independent flux determination from the model. The incorporation of this constraint is discussed further in Section 3.

At the time of the decision to acquire these data, it was deemed especially important to acquire $H$-band data because it was unknown whether the extrapolation from the (more routinely taken) $V / I$ data to $3.6 \mu \mathrm{m}$ would be feasible. In fact, in most cases, the OGLE $V$-band data did prove adequate to determine the $(I-[3.6])_{S}$ source color, but in five cases the source was either too red to obtain reliable $V$-band data or OGLE did not happen to observe the event in $V$ band when it was sufficiently magnified to determine $V-I$. In all but one of these cases (OGLE-2014-BLG-0337), the $H$-band data could be used to determine the source color (OGLE-2014-BLG-0805, OGLE-2014-BLG-0866, OGLE-2014-BLG-0944, OGLE2014-BLG-1021).

\subsection{Reductions}

With one exception the Spitzer data were reduced using the photometry tools available within MOPEX, a package designed to analyze IRAC data (Makovoz \& Marleau 2005): the analysis has been carried out with aperture photometry for six events (OGLE-2014-BLG-0099, OGLE-2014-BLG-0337, OGLE2014-BLG-0589, OGLE-2014-BLG-0805, OGLE-2014-BLG0944, and OGLE-2014-BLG-1021) and, to better deal with crowding, for all the remaining ones, with a point source response function (PRF) based photometry. ${ }^{58}$ The exception was OGLE-2014-BLG-1049, which was reduced using DoPhot (Schechter et al. 1993). All other light-curve data were reduced using image subtraction (Alard \& Lupton 1998). The CTIO $\mathrm{H}$ band data were reduced using DoPhot.

Error bars from each observatory were rescaled in order to impose $\chi^{2} /$ dof $\simeq 1$ based on the best-fit model.

\section{LIGHT-CURVE ANALYSIS}

The light curves were fitted to five-parameter models (plus two parameters for each observatory $i$, the source flux $F_{S, i}$, and the blended flux $\left.F_{B, i}\right)$,

$$
F_{i}(t)=F_{S, i} A_{i}\left(t ; t_{0}, u_{0}, t_{\mathrm{E}}, \pi_{\mathrm{E}, N}, \pi_{\mathrm{E}, E}\right)+F_{B, i}
$$

\footnotetext{
58 For a specific discussion of PRFs fitting in IRAC data we refer to the online manual for MOPEX http://irsa.ipac.caltech.edu/data/SPITZER/docs/ dataanalysistools/tools/mopex/mopexusersguide/.
} 
where (Paczyński 1986)

$$
\begin{aligned}
A_{i}\left(u_{i}\right) & =\frac{u_{i}^{2}+2}{\sqrt{u_{i}^{4}+4 u_{i}^{2}}} ; \quad u_{i}^{2} \equiv\left(\tau_{i}^{\prime}\right)^{2}+\left(\beta_{i}^{\prime}\right)^{2} ; \\
\tau^{\prime} & =\frac{t-t_{0}}{t_{\mathrm{E}}}+\Delta \tau_{i}(t) ; \quad \beta^{\prime}=u_{0}+\Delta \beta_{i}(t)
\end{aligned}
$$

and where, following closely the procedure based on a geocentric point of view outlined in Gould (2004), $\left(\Delta \tau_{i}(t)\right.$, $\left.\Delta \beta_{i}(t)\right)$ is the apparent lens-source offset in the Einstein ring relative to a uniform trajectory, as seen by the $i$ th observatory, due to the physical offset (in AU) of this observatory from a rectilinear trajectory defined by Earth's position and velocity vectors at the peak of the event, $\left(t_{0, \oplus}\right)$.

The physical offset of the observatory $\Delta \boldsymbol{p}_{i}(t)=\left(\Delta p_{i, N}(t), \Delta p_{i, E}(t)\right)$ is the sum of two terms

$$
\boldsymbol{p}_{i}(t)=\mathbf{s}(t)+\boldsymbol{t}_{i}(t) .
$$

The first term (common to all observatories) is the offset of the apparent position of the Sun (projected on the plane of the sky) relative to where it would be if Earth were in rectilinear motion (see Gould 2004, and specifically his Figure 2). The second term (called " $t$ " because it usually reflects so-called "terrestrial parallax," as opposed to the "orbital parallax") is the projected separation of Earth's center from the $i$ th observatory. Both terms are, by convention, scaled to $1 \mathrm{AU}$. The sign convention is due to the explicitly "geocentric" framework. For terrestrial observatories, for which we use Earth's ephemerides and the location of each observatory relative to Earth's center, $\left|\boldsymbol{t}_{i}\right| \ll 1$, although this term can in principle be important, particularly for high-magnification events (Gould 1997; Gould et al. 2009; Yee et al. 2009; Gould \& Yee 2013). For Spitzer $\left|\boldsymbol{t}_{i}\right| \sim \mathcal{O}(1)$, with the spacecraft position relative to Earth being available as a function of time from the Horizons Ephemeris System. ${ }^{59}$ Then, in analogy to Equation (8) of Gould (2004),

$$
\begin{aligned}
\Delta \tau_{i} & =-\frac{\Delta p_{i, N} \pi_{\mathrm{E}, N}+\Delta p_{i, E} \pi_{\mathrm{E}, E}}{\mathrm{AU}} \\
\Delta \beta_{i} & =-\frac{-\Delta p_{i, E} \pi_{\mathrm{E}, N}+\Delta p_{i, N} \pi_{\mathrm{E}, E}}{\mathrm{AU}} .
\end{aligned}
$$

As discussed in Section 2.4, for each event (except OGLE2014-BLG-0337) we measured the instrumental source color in either $(V-I)_{S}$ or $(I-H)_{S}$. We then determined the $(I-[3.6])_{S}$ color using a $V I$ [3.6] or $I H$ [3.6] color-color relation derived from field stars. These estimates typically have errors of $\sigma_{I-[3.6]}=0.06-0.1 \mathrm{mag}$, although they are larger in a few cases. These color measurements were then incorporated into the fit by

$$
\chi_{\text {color }}^{2}=\frac{\left[(I-[3.6])-2.5 \log \left(F_{S, \text { Spitzer }} / F_{S, \text { OGLE }}\right)\right]^{2}}{\sigma_{I-[3.6]}^{2}} .
$$

In most cases inclusion of this term made almost no difference, generally because the fit values of $F_{S, \text { Spitzer }}$ and $F_{S, \text { OGLE }}$ already yielded an $(I-[3.6])_{S}$ color that was consistent with the one derived from the color-color diagram. However, in a few cases,

\footnotetext{
59 http://ssd.jpl.nasa.gov/horizons
}

particularly when the Spitzer observations covered only a fragment of the Paczyński (1986) curve, this constraint proved to be important.

To locate the four solutions (with different parallax vectors $\pi_{\mathrm{E}}$ ) that are predicted from theory (Refsdal 1966; Gould 1994), we begin by fitting the ground-based light curve to the standard Paczyński (1986) three parameters $\left(t_{0, \oplus}, u_{0, \oplus}, t_{\mathrm{E}}\right)$, i.e., without parallax. We then add in Spitzer data and include two additional parameters $\pi_{\mathrm{E}}$ and apply Newton's method (Simpson 1740, p. 81). This quickly locates the $\Delta u_{0,-,+}$ solution. We then reverse the signs of $\left(u_{0}, \pi_{\mathrm{E}, N}\right)$ (Skowron et al. 2011) and again apply Newton's method, which locates the $\Delta u_{0,-,-}$ solution. We then take the original solution, put in a large value for $\pi_{\mathrm{E}, N}$, and apply Newton's method, which locates the $\Delta u_{0,+,+}$ solution, and finally we reverse the signs of $\left(u_{0}, \pi_{\mathrm{E}, N}\right)$ for this solution and again apply Newton's method to obtain the fourth solution.

The only event for which this procedure failed was OGLE2014-BLG-1049. The reason for the failure is that the event was high magnification as seen from Earth $\left(u_{0, \oplus}<0.01\right)$ and was also high magnification as seen from Spitzer. However, because the first Spitzer point was 1 day after peak, $u_{0, \text { Spitzer }}$ is consistent with both zero and values that are significantly larger than $u_{0, \oplus}$. These characteristics lead to a merger of the two solutions $\Delta u_{0, \pm,+}$ and also a merger of the solutions $\Delta u_{0, \pm,-}$. Nevertheless, although the merged solutions are unstable to Newton's method, they have quite well behaved minima and constitute an interesting limiting case of the standard fourfold degeneracy.

Table 2 lists the fitted parameters for each of the four solutions for each of the 21 events. The $\Delta \chi^{2}$ offset between each of the other three solutions and the best one is shown in the second column.

An additional analysis we might in principle address is related to the determination of the parallax from ground-based data alone. While formally it is extremely straightforward to fit the light curves after excluding the Spitzer data (and indeed, within our fit procedure, the effect of parallax for ground-based data from orbital motion is automatically included), historical experience with ground-based parallax measurements shows that a more cautious approach is required. In contrast to spacebased parallaxes, in which the signal derives from obvious differences in the peaks of the event as seen from wellseparated observatories, ground-based parallaxes derive from subtle distortions of the light curves. These can be caused or corrupted by "xallarap" (binary motion of the source during the event), very small distortions due to unrecognized binary lenses, or just systematics in the data. These problems can be mitigated by the presence of well-understood structures in the light curve for events that contain a planet (e.g., Muraki et al. 2011), but for point-lens events, which are otherwise featureless, ground-based parallaxes are especially prone to such corruption. Indeed, in the only systematic study of pointlens ground-based parallaxes (Poindexter et al. 2005), even within a restricted sample of parallax detections with $\Delta \chi^{2}>100$, there was a strong evidence for xallarap in $23 \%$ of cases. As described in some detail by Poindexter et al. (2005), the tests for xallarap (and related systematics) are quite involved and are well beyond the scope of the present work, which relies on much more straightforward space-based parallaxes. 
(a)

OGLE-2014-BLG-0099: $\Delta \chi^{2}=17.33,0,241.54,202.96(-+,--,++,+-)$
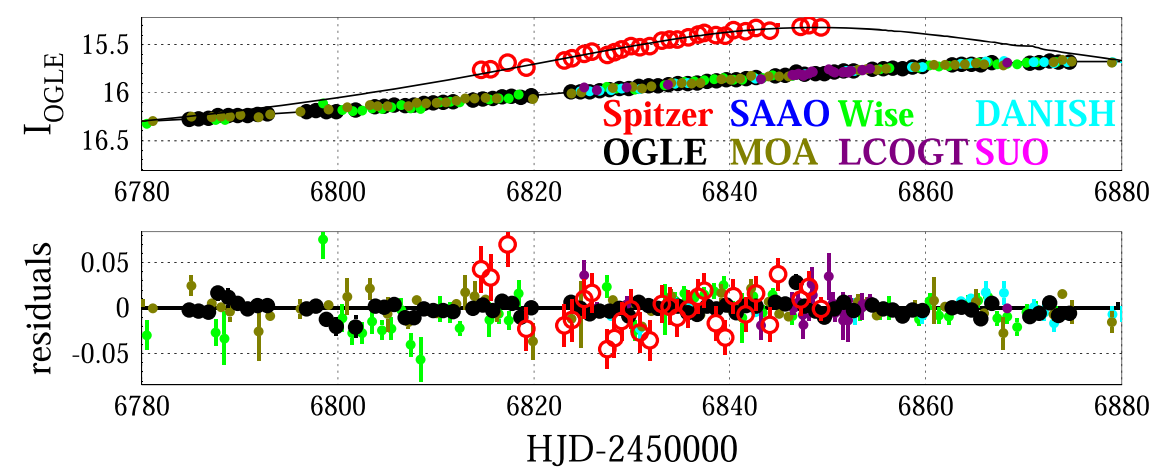

\section{Heliocentric velocity $(\mathrm{km} / \mathrm{s}) \quad$ Geocentric parallax}
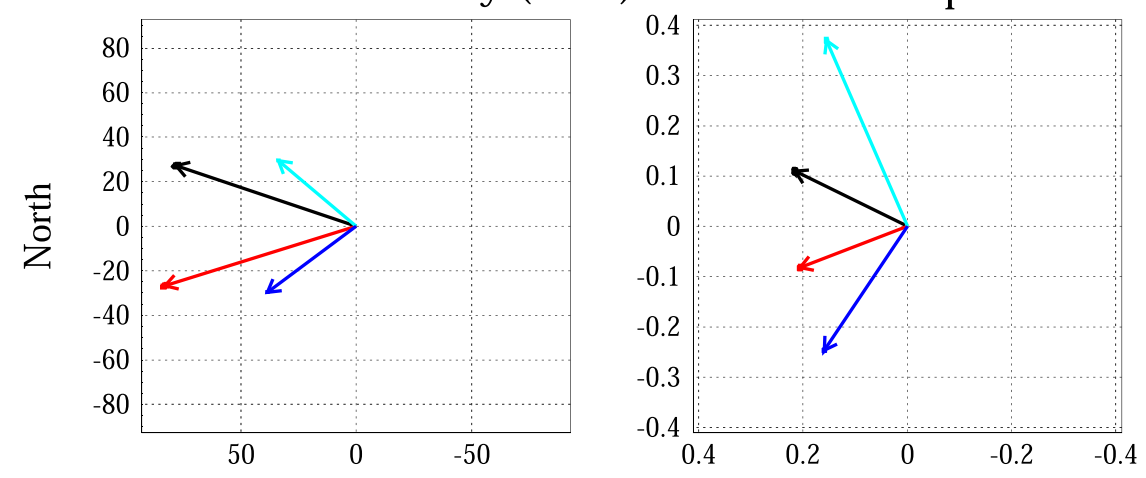

East

Figure 1. (a) OGLE-2014-BLG-0099. Top: light-curve data together with the Spitzer and the ground-based best-fit models. Second panel from top: residual light curve. In both panels the Spitzer and the ground-based data are shown as empty and filled circles, respectively. For purposes of display, all the data sets are binned with 1 point per epoch. The color codes are indicated in the top panel: red, black, blue, olive green, green and purple for Spitzer, OGLE, SAAO (PLANET), MOA, Wise, LCOGT (RoboNet; the details of the different telescopes of the network used are given in Table 1) and for the MiNDSTEp collaboration the Danish (cyan) and the Salerno University Telescope (magenta). In the two bottom panels we show the projected heliocentric velocity $\tilde{v}_{\text {hel }}$ (left) and the geocentric parallax vectors $\pi_{\mathrm{E}, \mathrm{geo}}$ and ellipse errors (which can, however, be too small to be seen), in the northeast equatorial frame, as given in Table 2. The values of the $\Delta \chi^{2}$, as reported in the title, are color-coded as black, red, cyan and blue, from the best solution to the worst. (b) OGLE-2014-BLG-0115. Panels and symbols as in (a). (c) OGLE-2014-BLG-0337. Panels and symbols as in (a). (d) OGLE-2014-BLG-0419. Panels and symbols as in (a). (e) OGLE-2014-BLG-0494. Panels and symbols as in (a). (f) OGLE-2014BLG-0589. Panels and symbols as in (a). (g) OGLE-2014-BLG-0641. Panels and symbols as in (a). (h) OGLE-2014-BLG-0667. Panels and symbols as in (a). (i) OGLE-2014-BLG-0670. Panels and symbols as in (a). (j) OGLE-2014-BLG-0678. Panels and symbols as in (a). (k) OGLE-2014-BLG-0752. Panels and symbols as in (a). (1) OGLE-2014-BLG-0772. Panels and symbols as in (a). (m) OGLE-2014-BLG-0805. Panels and symbols as in (a). (n) OGLE-2014-BLG-0807. Panels and symbols as in (a). (o) OGLE-2014-BLG-0866. Panels and symbols as in (a). (p) OGLE-2014-BLG-0874. Panels and symbols as in (a). (q) OGLE-2014-BLG-0939. Panels and symbols as in (a). (r) OGLE-2014-BLG-0944. Panels and symbols as in (a). (s) OGLE-2014-BLG-0979. Panels and symbols as in (a). (t) OGLE-2014BLG-1021. Panels and symbols as in (a). (u) OGLE-2014-BLG-1147. Panels and symbols as in (a).

\section{VISUAL REPRESENTATIONS OF SOLUTIONS}

Figure 1 gives a visual representation of all the key information for 20 of the 21 events (except OGLE-2014BLG-1049). For each event, the upper panel shows the lightcurve data from all observatories. All have been aligned to OGLE fluxes (and then converted to OGLE magnitudes) in the standard fashion. That is,

$$
F_{\mathrm{OGLE}, \mathrm{sys}}=\left(F-F_{B, i}\right) \frac{F_{S, \mathrm{OGLE}}}{F_{S, i}}+F_{B, \mathrm{OGLE}},
$$

where the $F_{S}$ and $F_{B}$ are determined from the fit. This panel also shows the model(s), i.e., the model light curve as seen from Earth and from Spitzer. Note that the model is extended beyond the range of Spitzer observations although Spitzer could not actually observe the events at these times due to Sunangle restrictions. The $\Delta \chi^{2}$ values for the four solutions are listed above this panel, always in the same order $(-+,--,++,+$ $-)$. The next panel shows the residuals.

The lower two panels show two different representations of the four parallax solutions. In each case, the solutions are color coded in order of increasing $\chi^{2}$, namely, black, red, cyan, and blue. The right panel shows the $\pi_{\mathrm{E}, \text { geo }}$ vectors and error ellipses in the geocentric frame, i.e., those that are directly returned by the fit. As described below, the $\pi_{\mathrm{E} \text {,hel }}$ vectors would have exactly the same lengths but slightly different directions compared to the $\pi_{\mathrm{E} \text {,geo }}$ vectors that are shown.

In the left panel, we show the heliocentric projected velocities $\tilde{v}_{\text {hel }}$, defined as

$$
\tilde{\boldsymbol{v}}_{\text {hel }}=\tilde{\boldsymbol{v}}_{\mathrm{geo}}+\boldsymbol{v}_{\oplus, \perp} ; \quad \tilde{\boldsymbol{v}}_{\mathrm{geo}}=\frac{\pi_{\mathrm{E}, \mathrm{geo}}}{\pi_{\mathrm{E}}^{2}} \frac{\mathrm{AU}}{t_{\mathrm{E}, \mathrm{geo}}},
$$

where $\boldsymbol{v}_{\oplus, \perp}$ is the velocity of Earth projected on the plane of the sky and evaluated at $t_{0, \oplus}$. While this quantity varies slightly from event to event in the sample, most are quite close to 
(b)
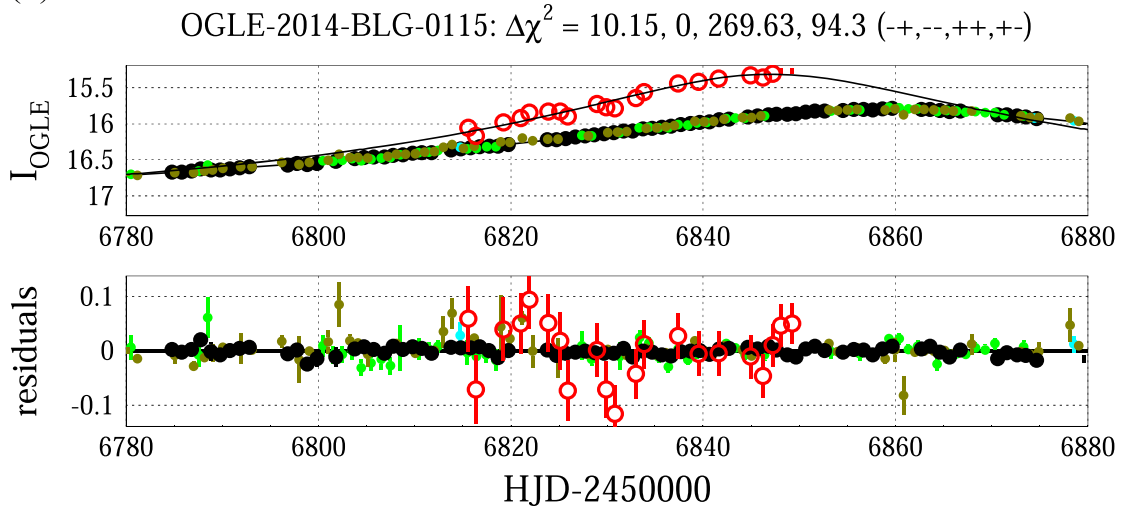

Heliocentric velocity $(\mathrm{km} / \mathrm{s}) \quad$ Geocentric parallax

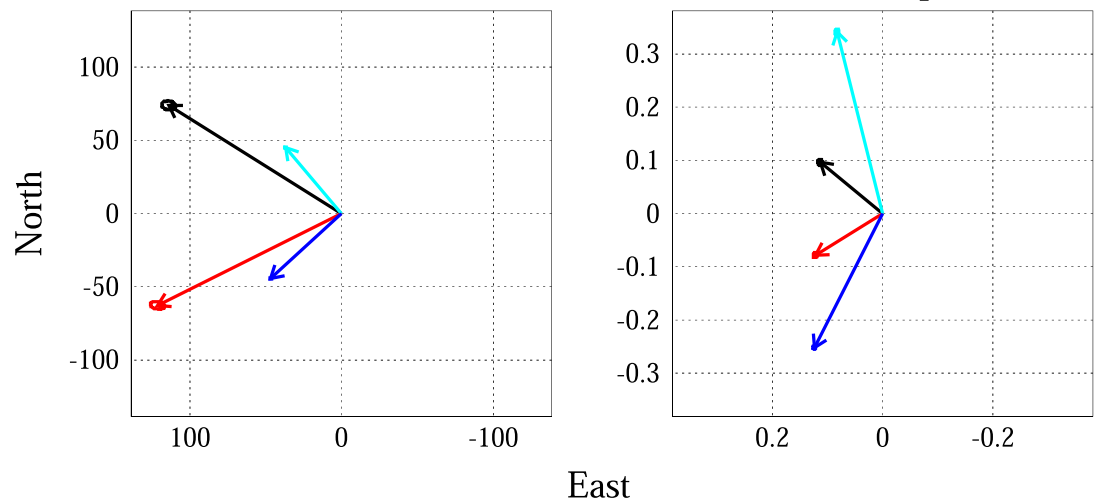

(c)
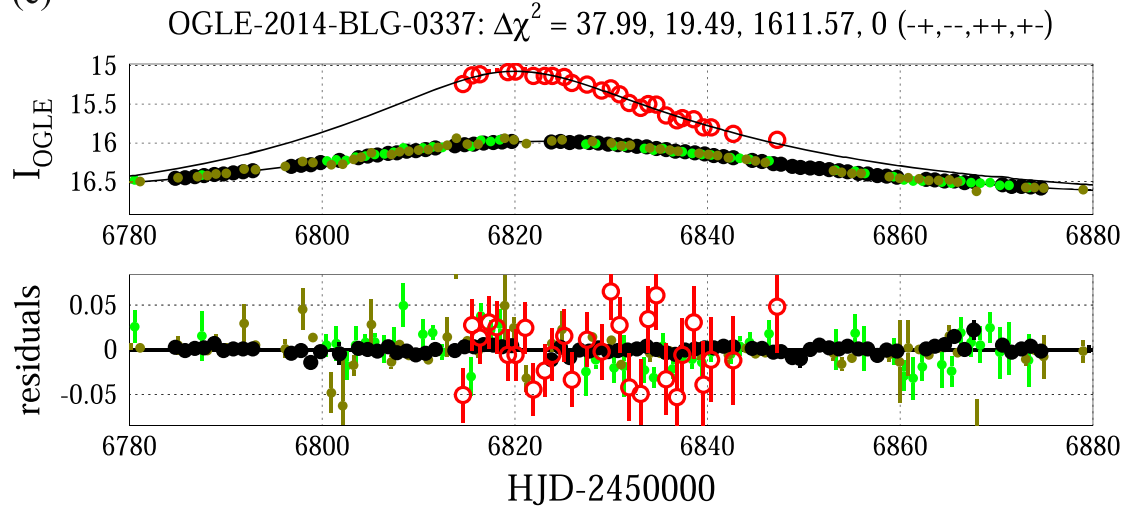

Heliocentric velocity $(\mathrm{km} / \mathrm{s}) \quad$ Geocentric parallax
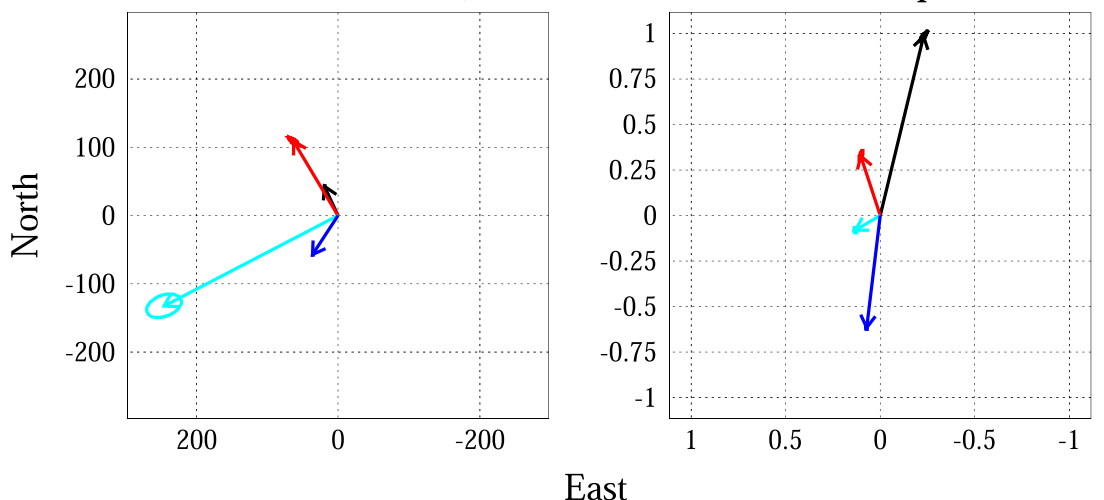

Figure 1. (Continued.) 
(d)
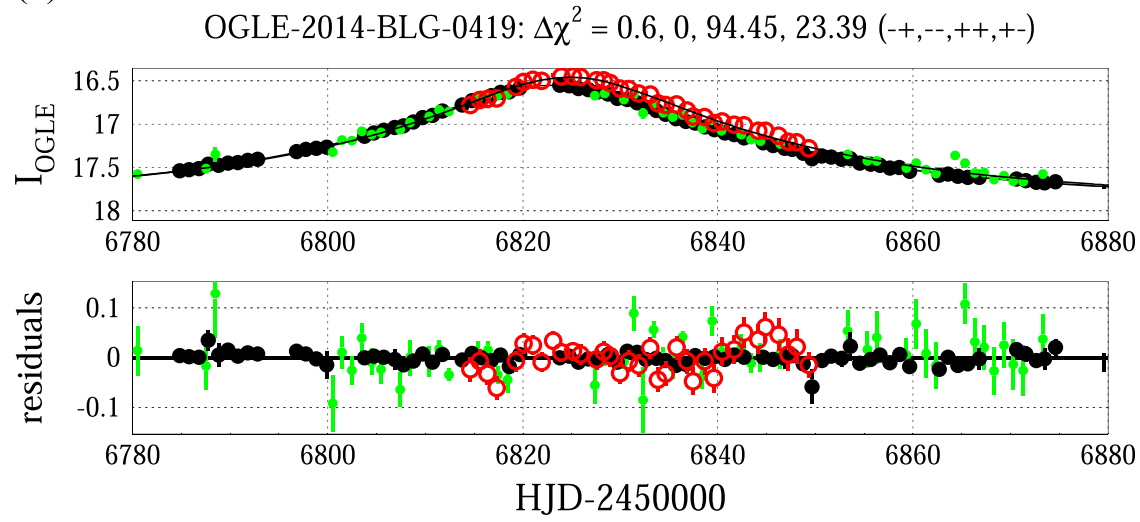

\section{Heliocentric velocity $(\mathrm{km} / \mathrm{s}) \quad$ Geocentric parallax}
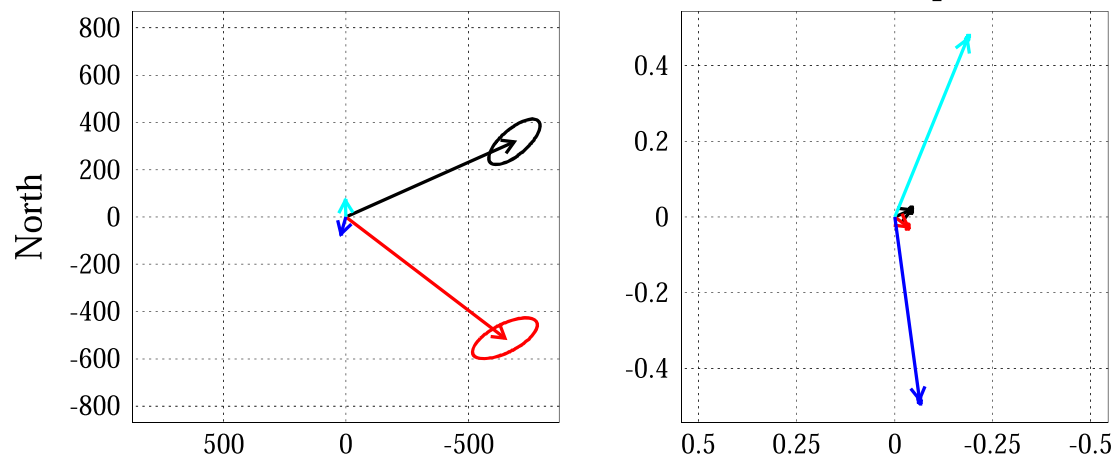

(e)

East
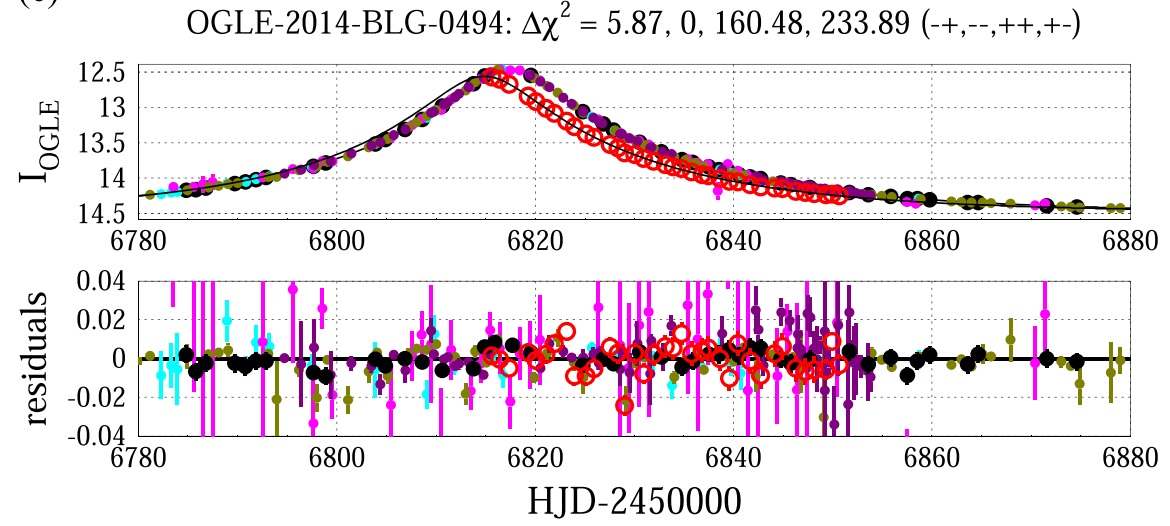

\section{Heliocentric velocity $(\mathrm{km} / \mathrm{s}) \quad$ Geocentric parallax}
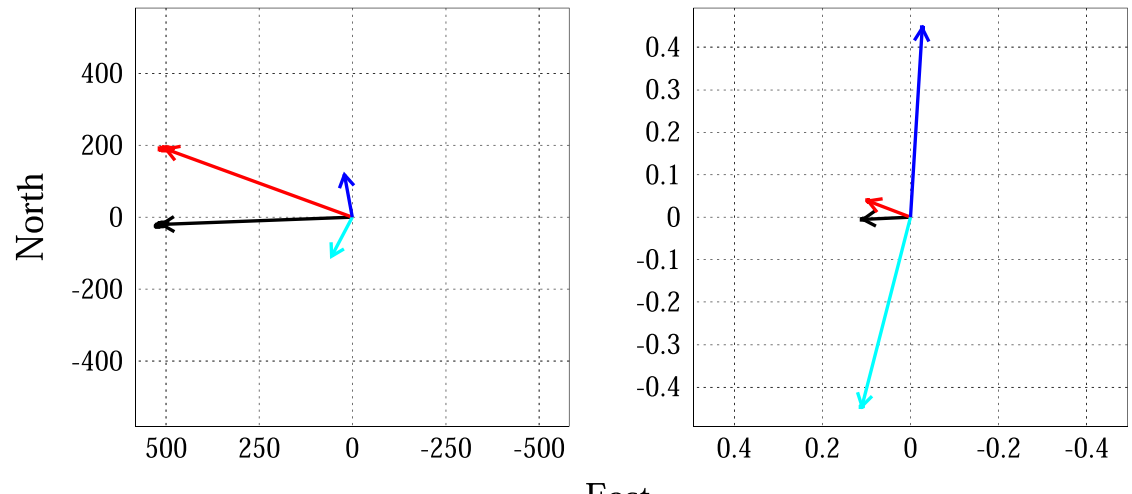

East

Figure 1. (Continued.) 
(f)
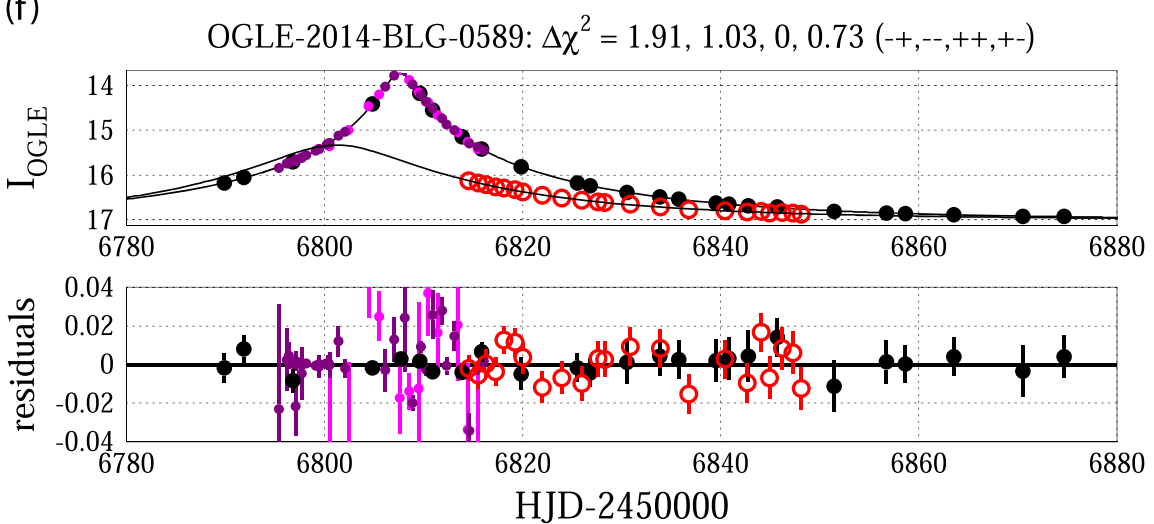

Heliocentric velocity $(\mathrm{km} / \mathrm{s}) \quad$ Geocentric parallax
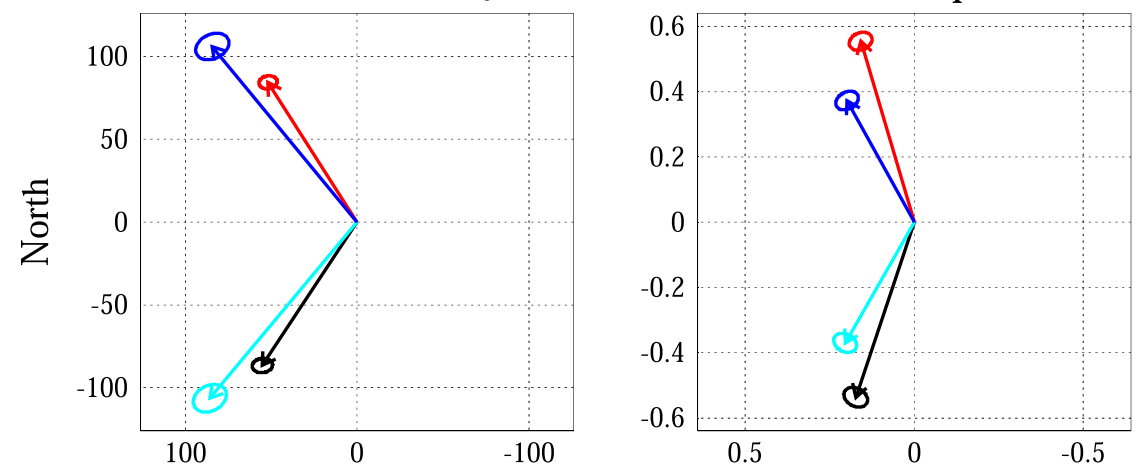

(g)

East
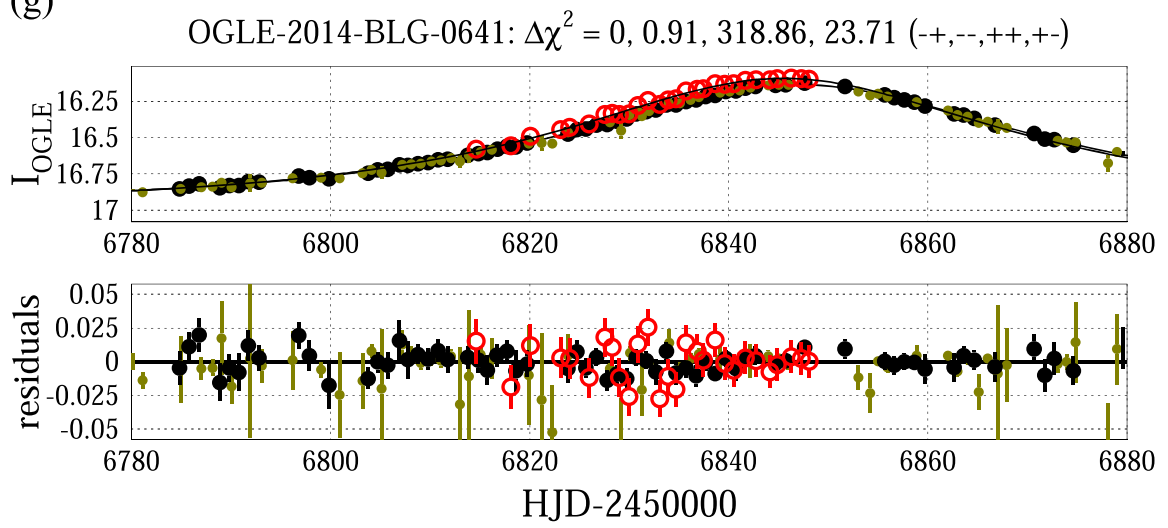

Heliocentric velocity $(\mathrm{km} / \mathrm{s}) \quad$ Geocentric parallax
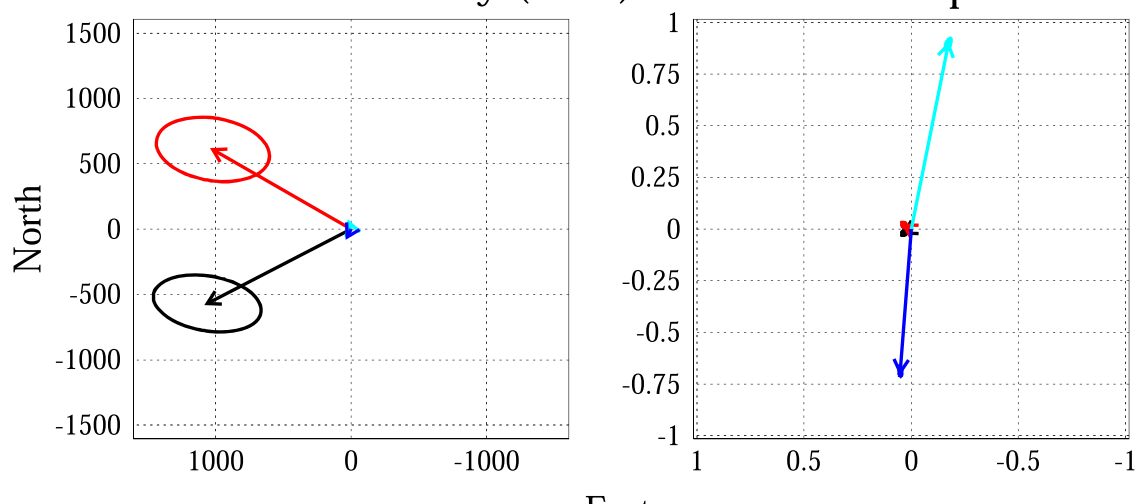

East

Figure 1. (Continued.) 
(h)
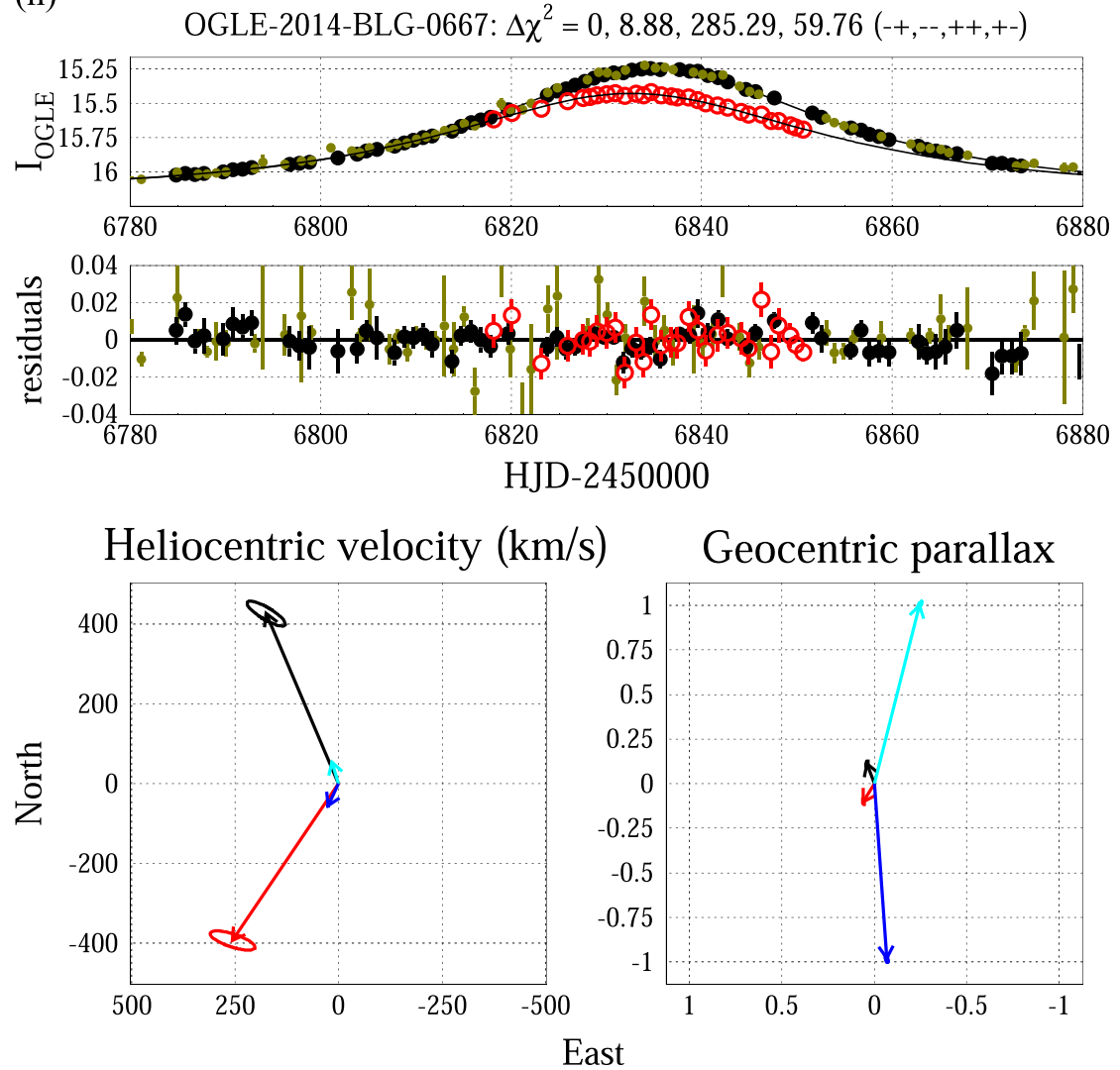

(i)

OGLE-2014-BLG-0670: $\Delta \chi^{2}=3.18,0,5.15,32.74(-+,--,++,+-)$
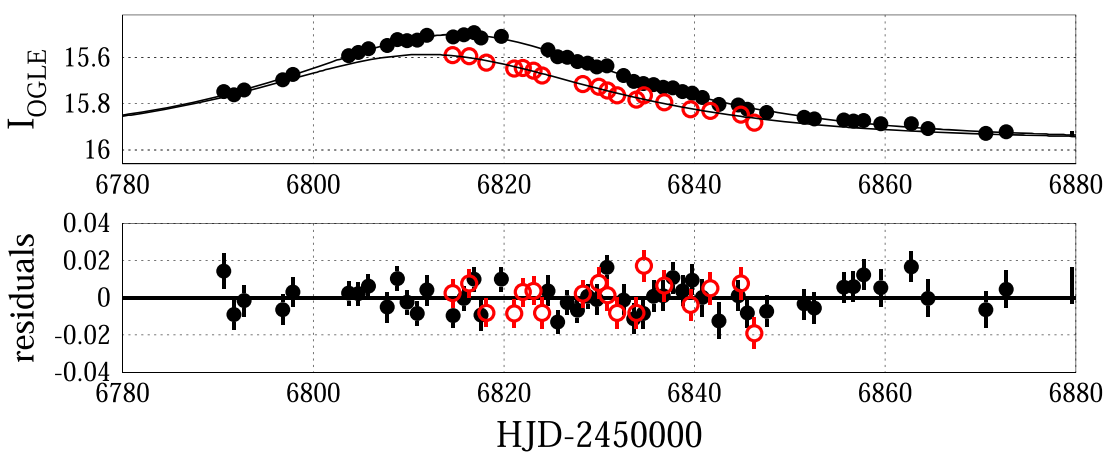

Heliocentric velocity $(\mathrm{km} / \mathrm{s}) \quad$ Geocentric parallax
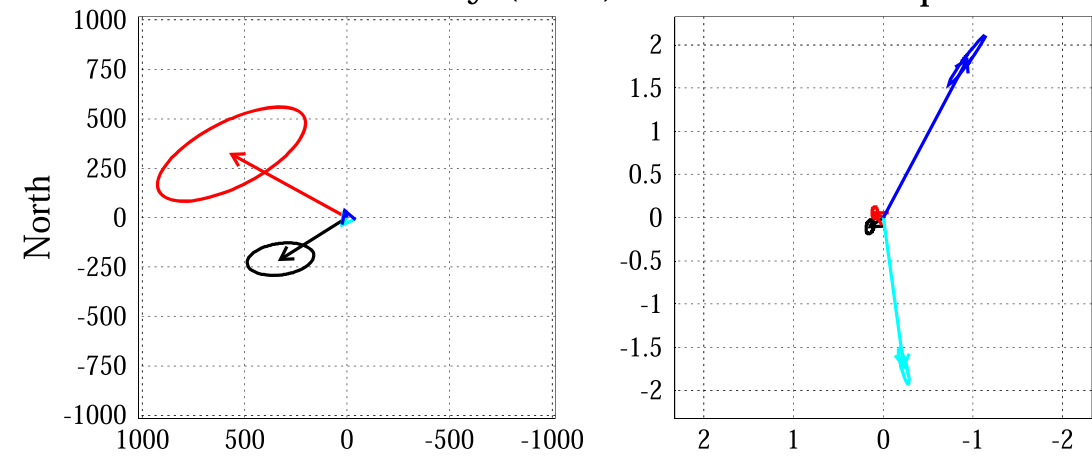

East

Figure 1. (Continued.) 
(j)

OGLE-2014-BLG-0678: $\Delta \chi^{2}=0.47,0,24.57,3.73(-+,--,++,+-)$
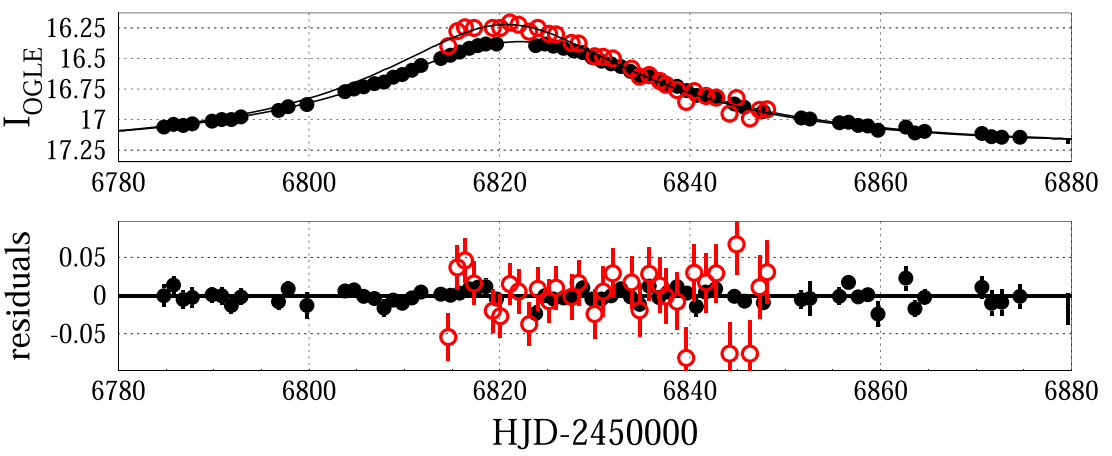

Heliocentric velocity $(\mathrm{km} / \mathrm{s}) \quad$ Geocentric parallax
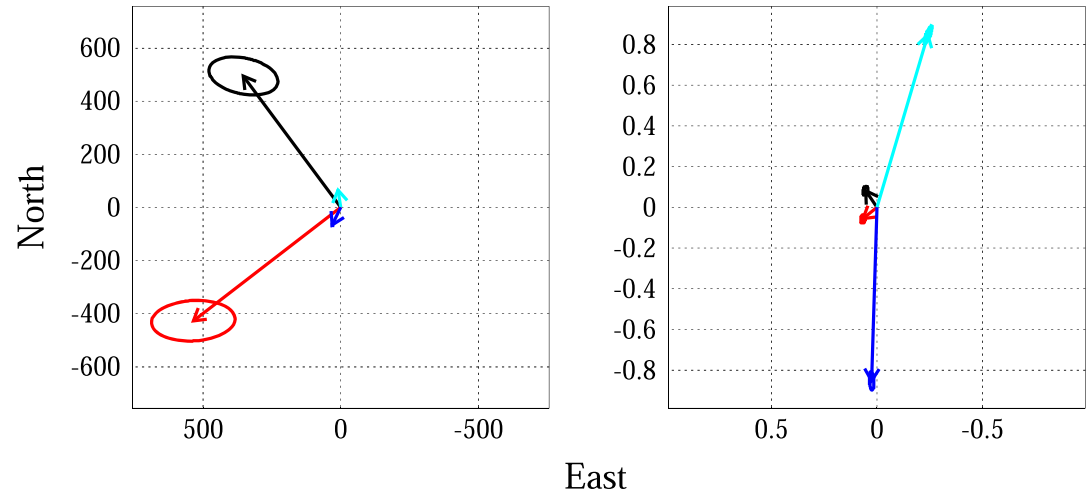

(k)

East

OGLE-2014-BLG-0752: $\Delta \chi^{2}=0.8,0,137.11,11.81(-+,--,++,+-)$
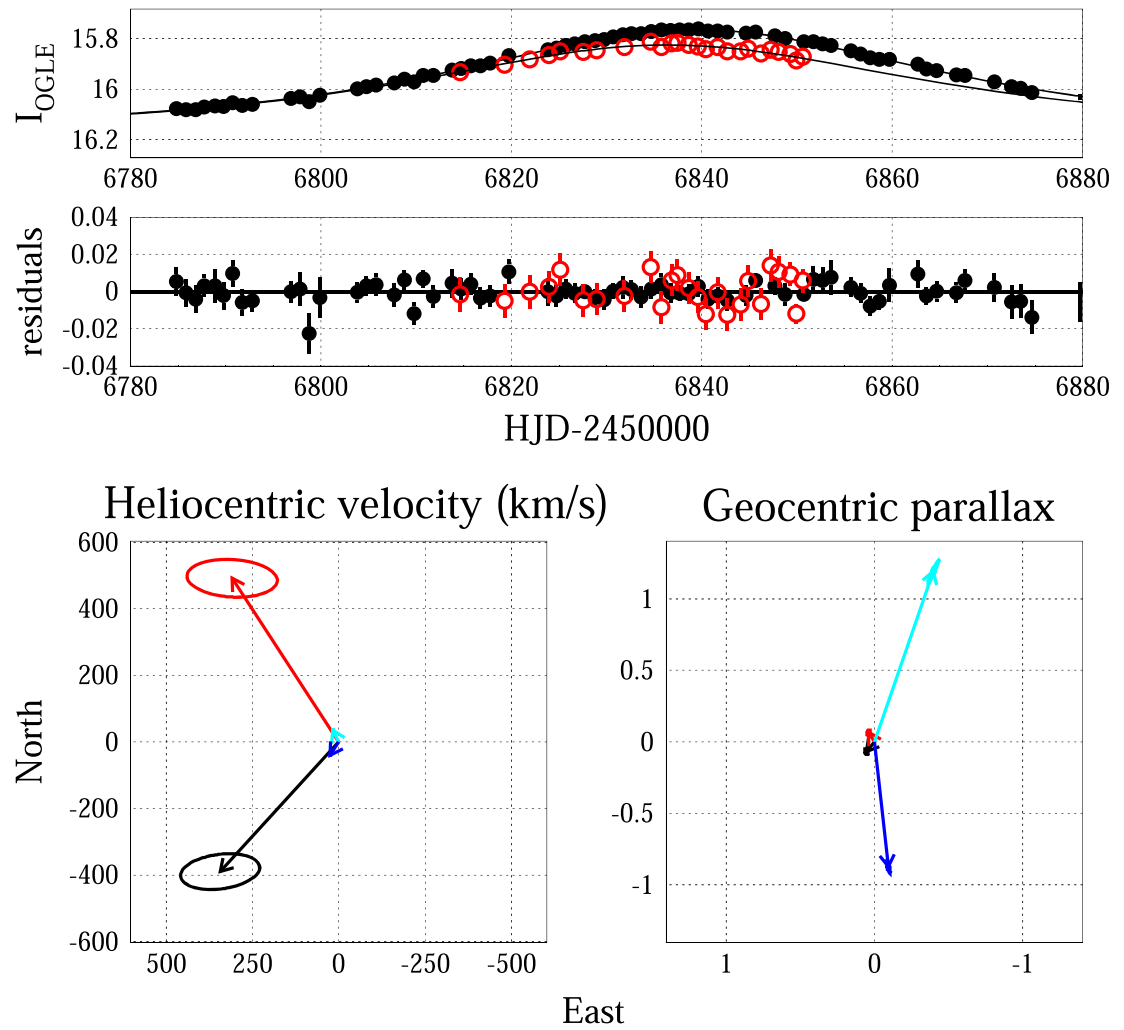

Figure 1. (Continued.) 
(1)

OGLE-2014-BLG-0772: $\Delta \chi^{2}=7.15,7.19,0,1.57(-+,--,++,+-)$
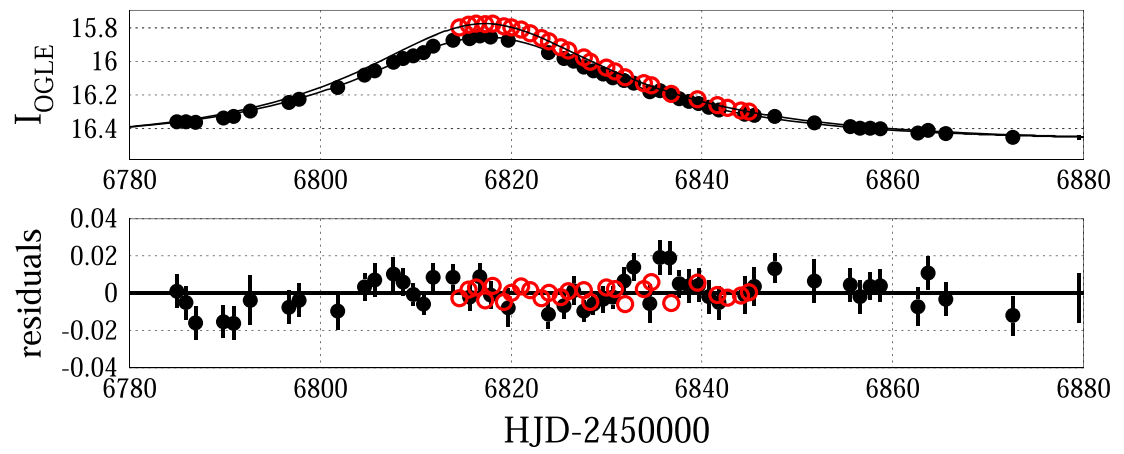

Heliocentric velocity $(\mathrm{km} / \mathrm{s}) \quad$ Geocentric parallax
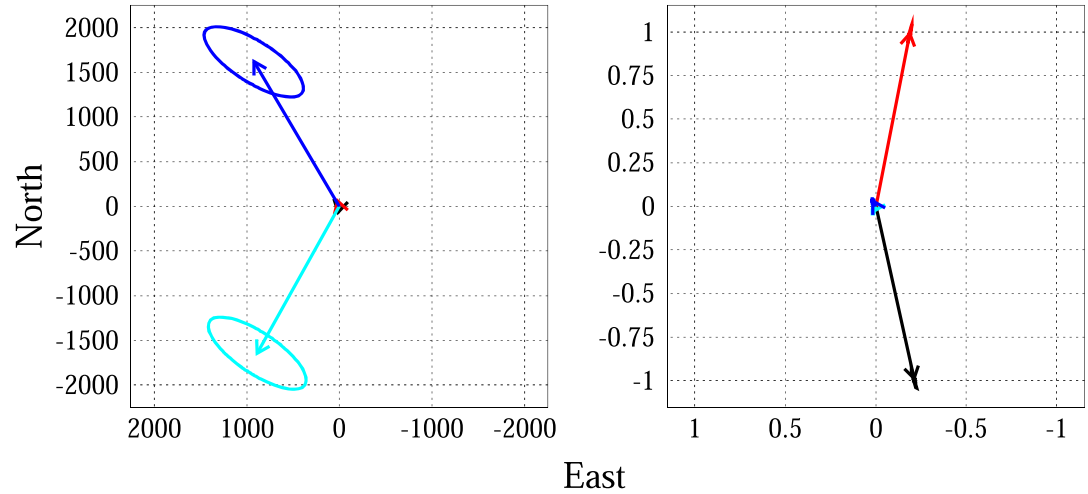

(m)

East
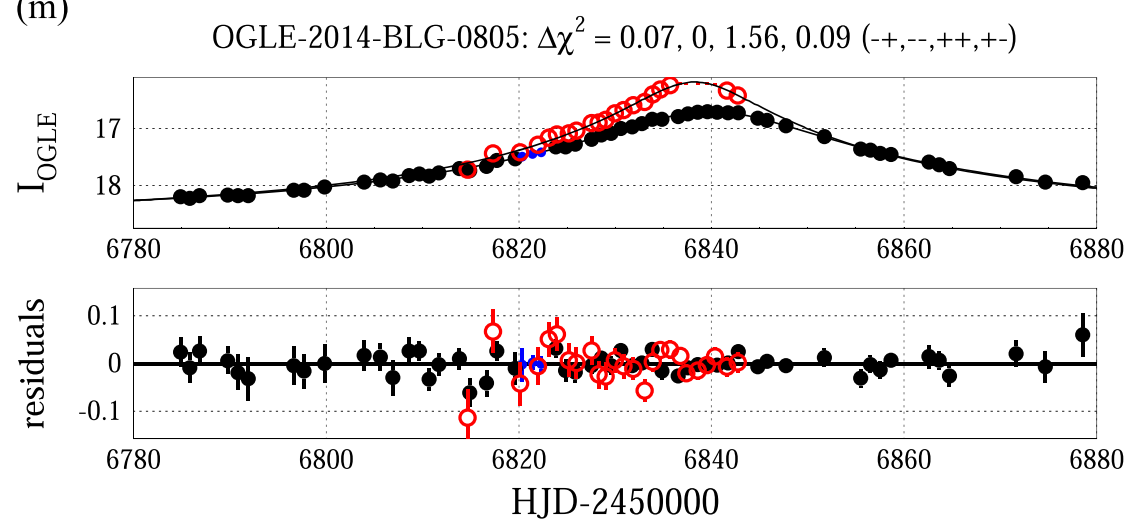

Heliocentric velocity $(\mathrm{km} / \mathrm{s}) \quad$ Geocentric parallax
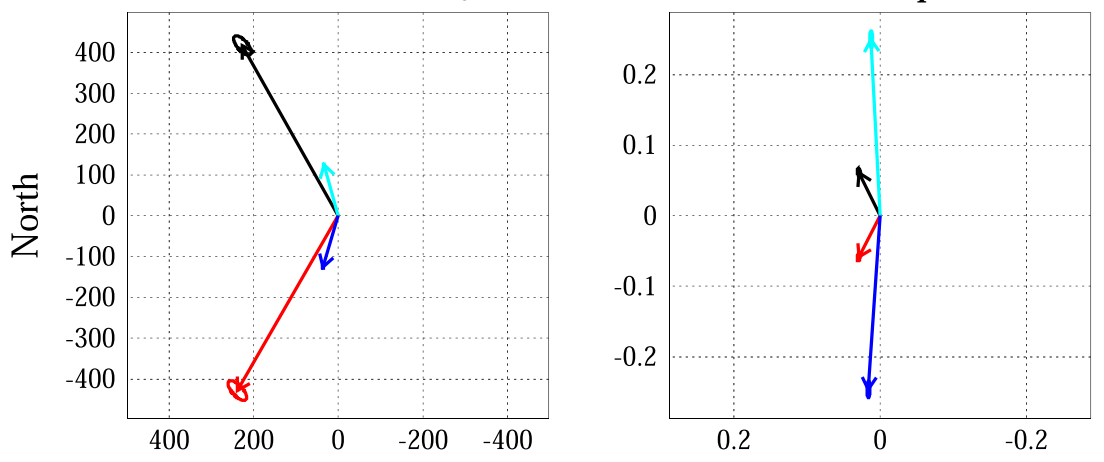

East

Figure 1. (Continued.) 
(n)
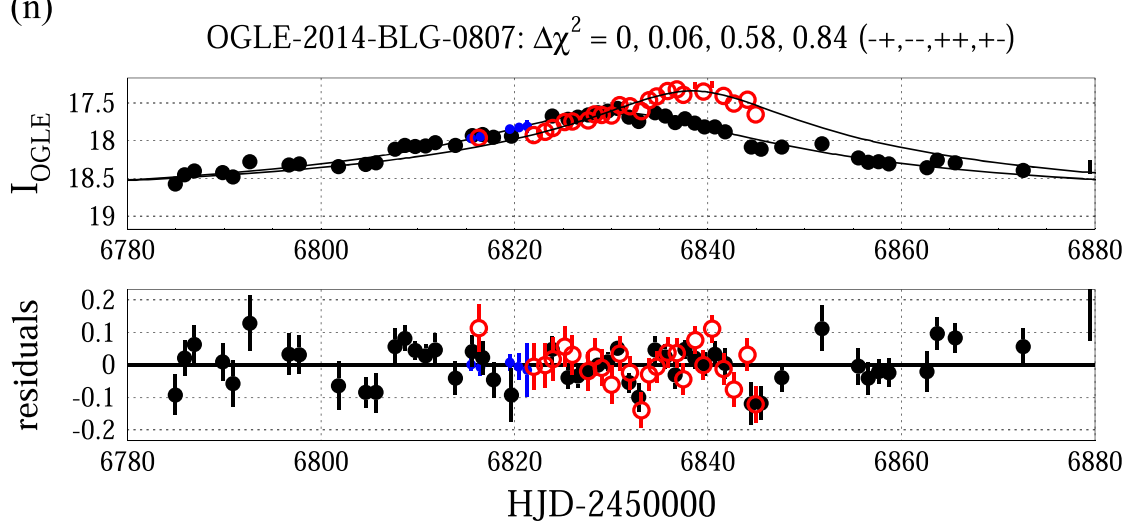

Heliocentric velocity $(\mathrm{km} / \mathrm{s}) \quad$ Geocentric parallax
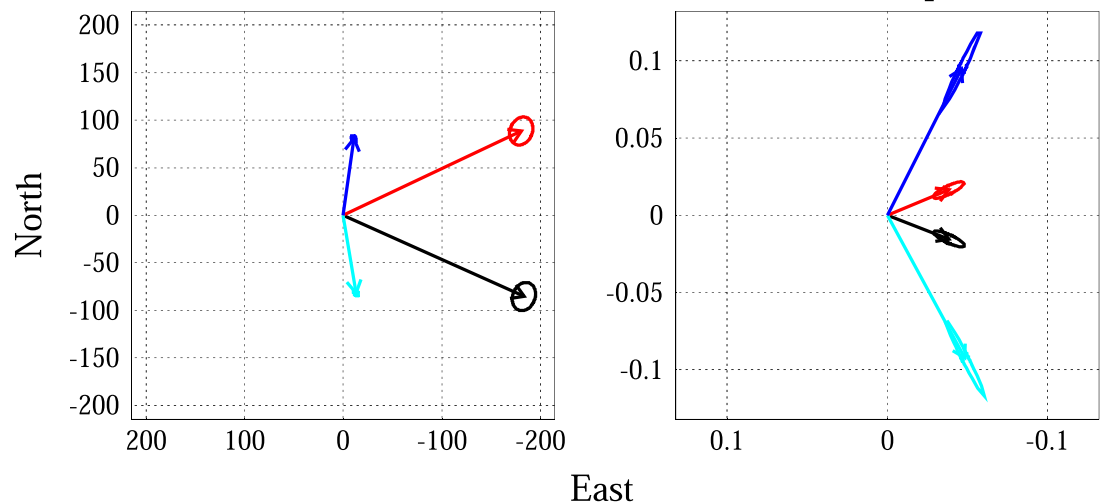

(o)
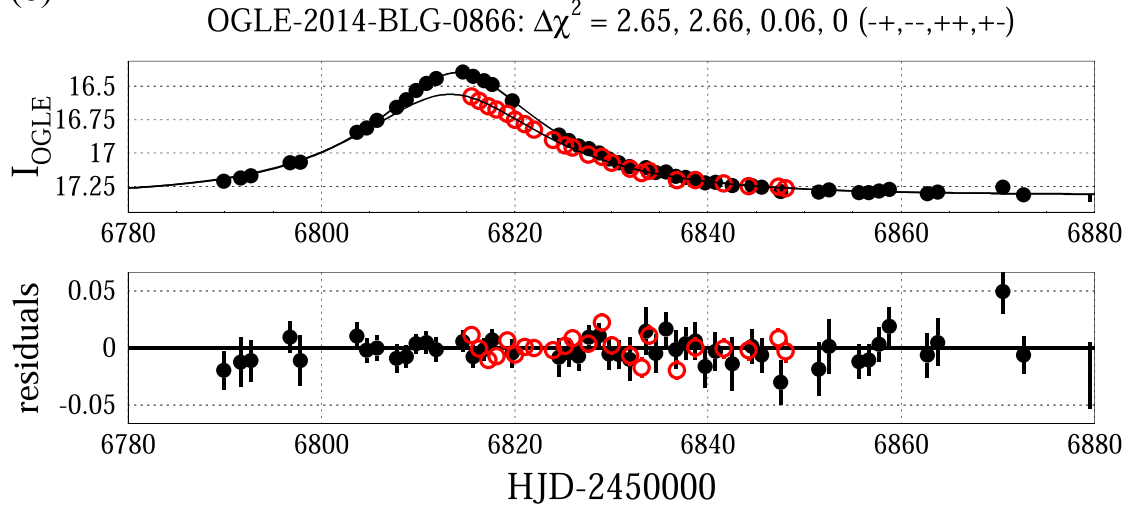

Heliocentric velocity $(\mathrm{km} / \mathrm{s}) \quad$ Geocentric parallax
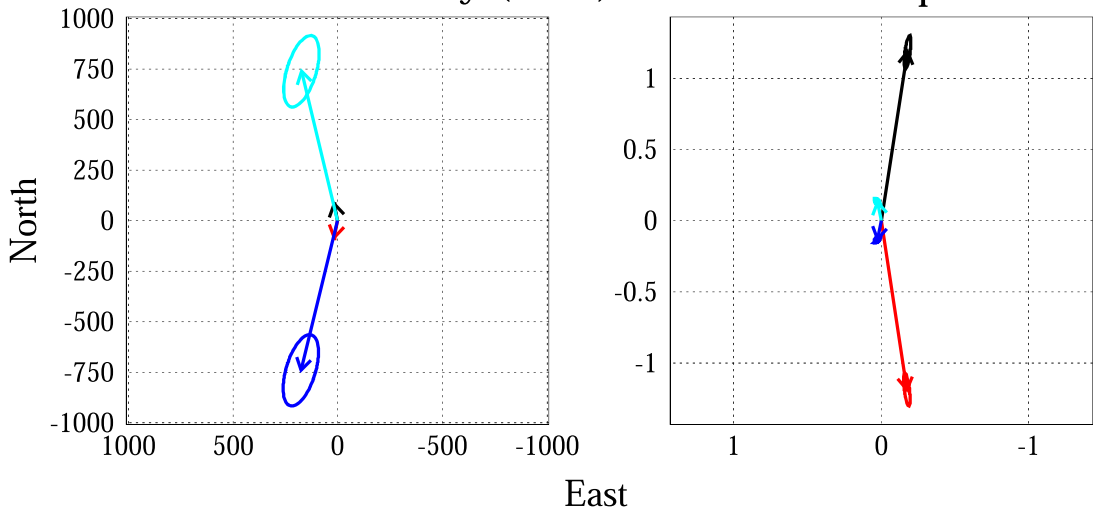

Figure 1. (Continued.) 
(p)

OGLE-2014-BLG-0874: $\Delta \chi^{2}=19.71,3.08,36.41,0(-+,--,++,+-)$
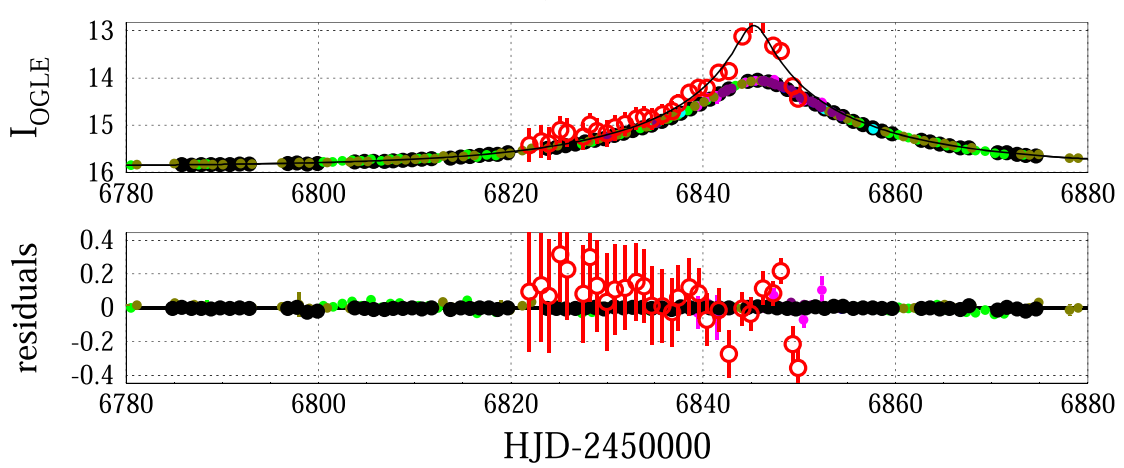

Heliocentric velocity $(\mathrm{km} / \mathrm{s}) \quad$ Geocentric parallax
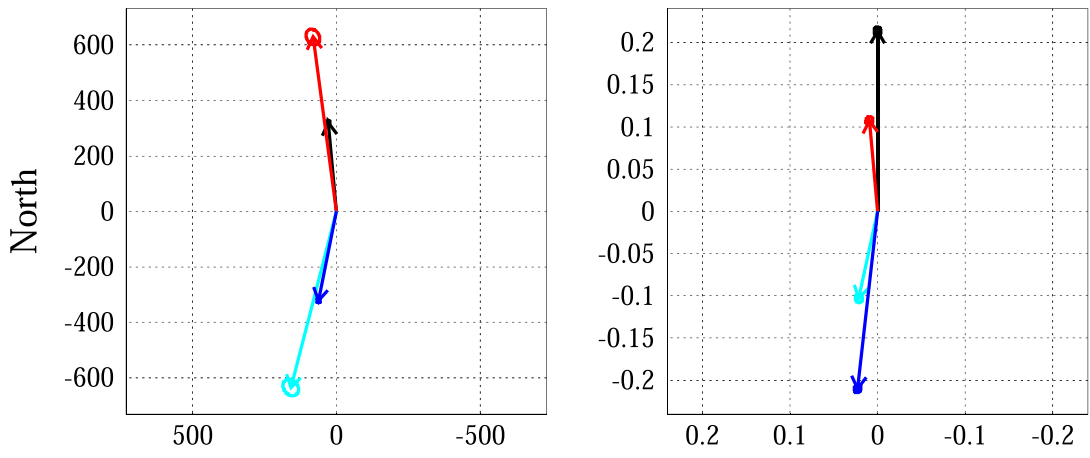

East

(q)
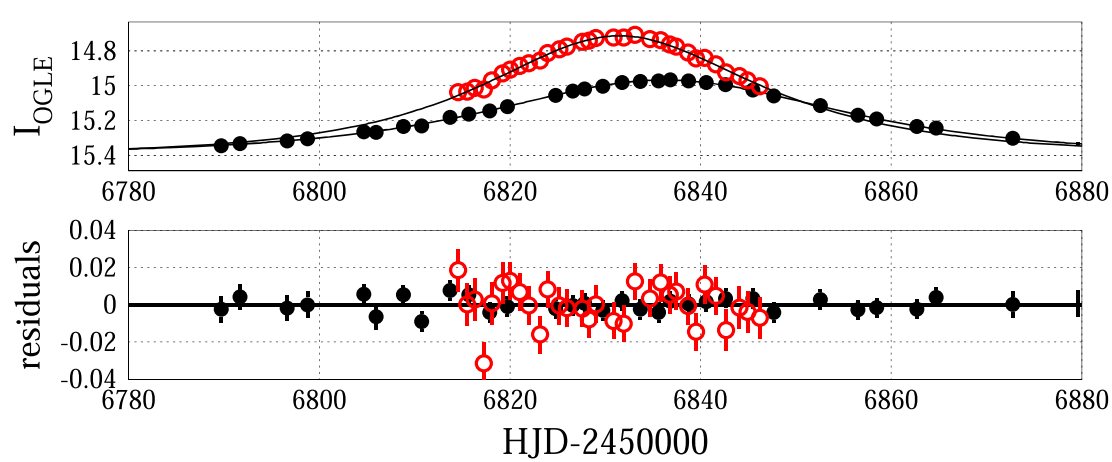

\section{Heliocentric velocity $(\mathrm{km} / \mathrm{s}) \quad$ Geocentric parallax}
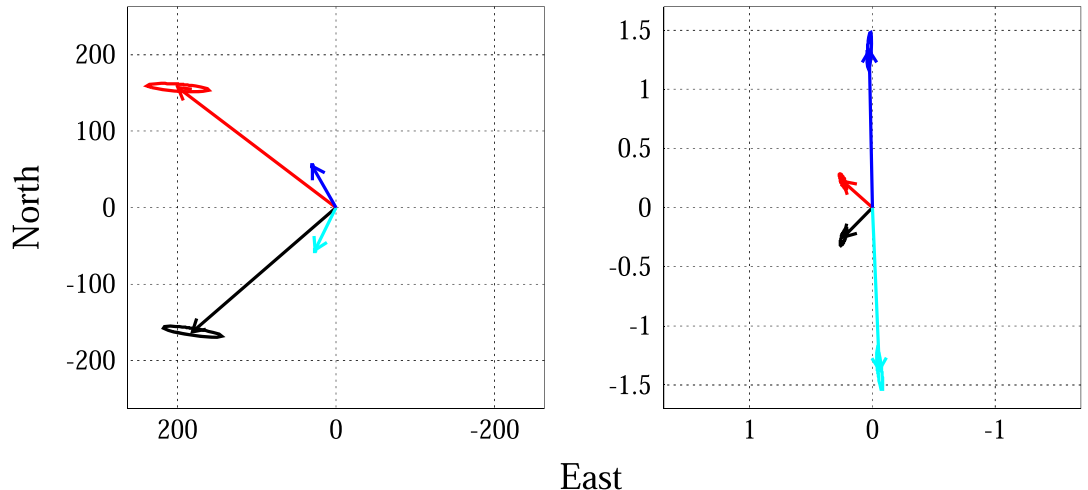

East

Figure 1. (Continued.) 
(r)
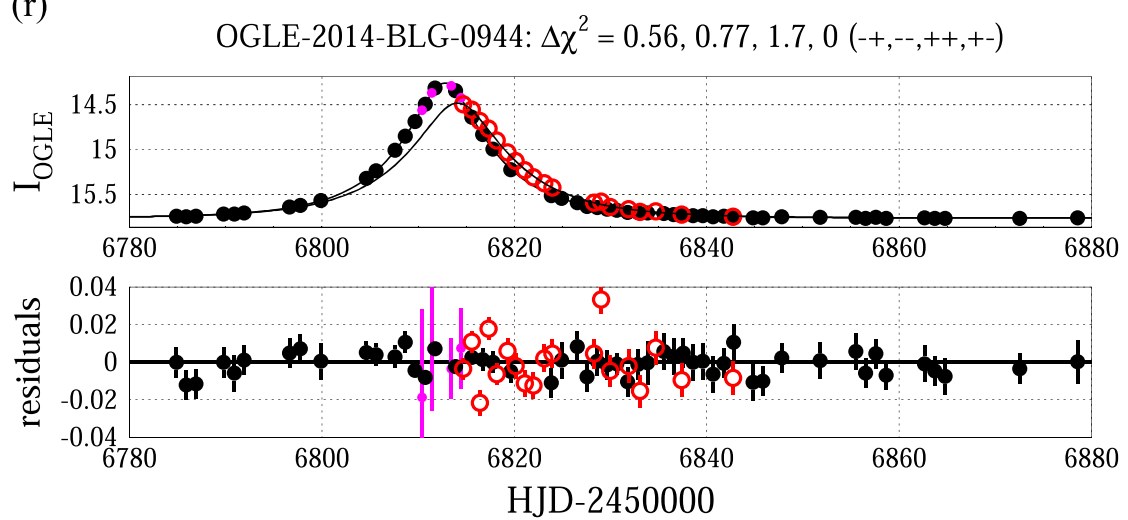

Heliocentric velocity $(\mathrm{km} / \mathrm{s}) \quad$ Geocentric parallax
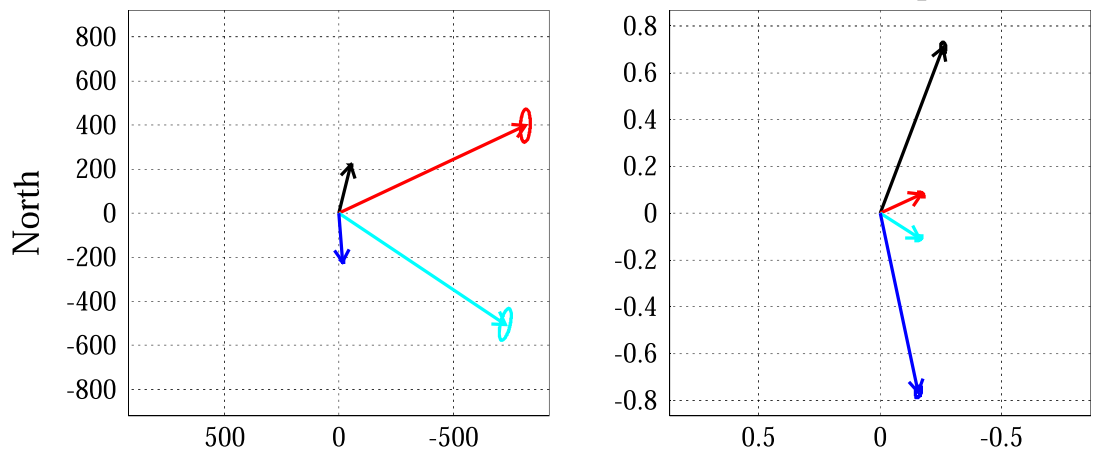

East

(s)
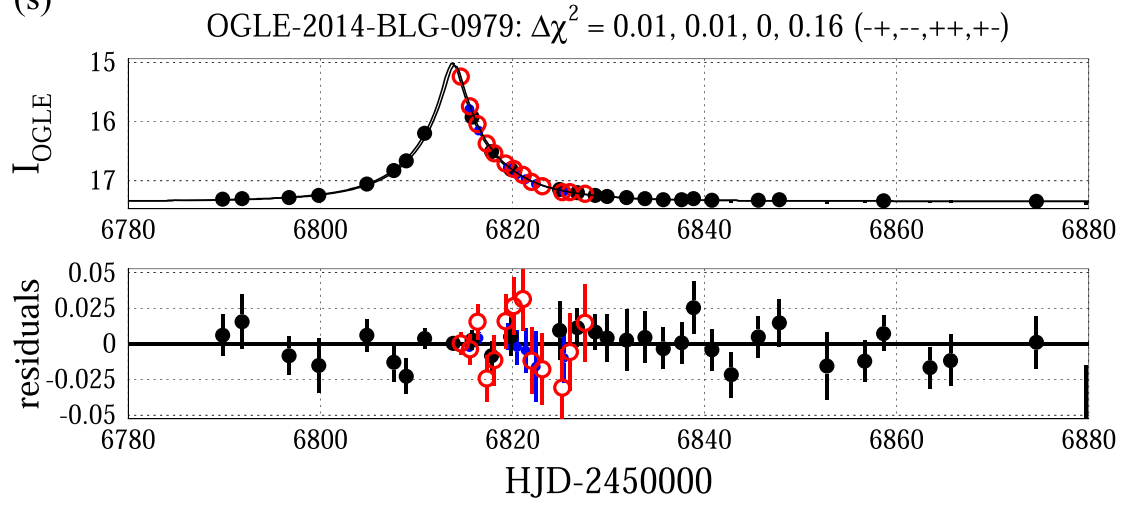

Heliocentric velocity $(\mathrm{km} / \mathrm{s}) \quad$ Geocentric parallax
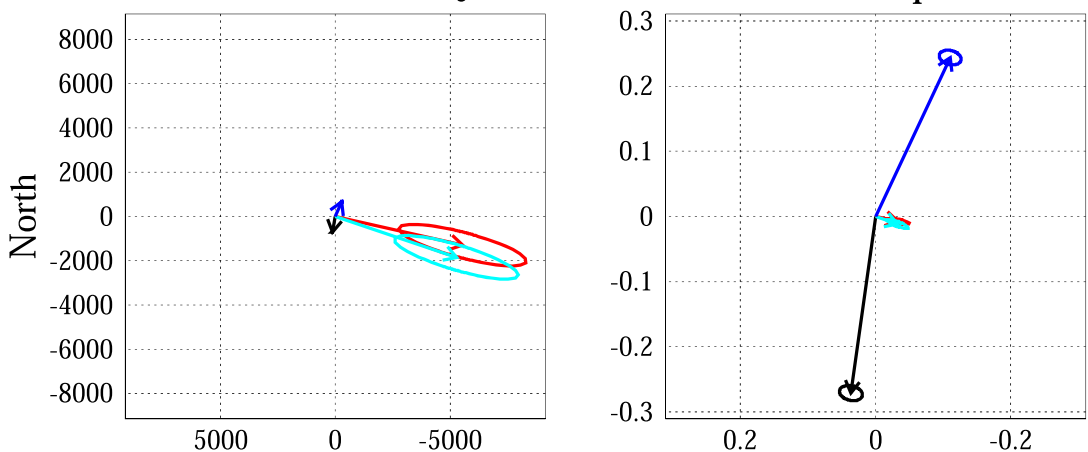

East

Figure 1. (Continued.) 
(t)
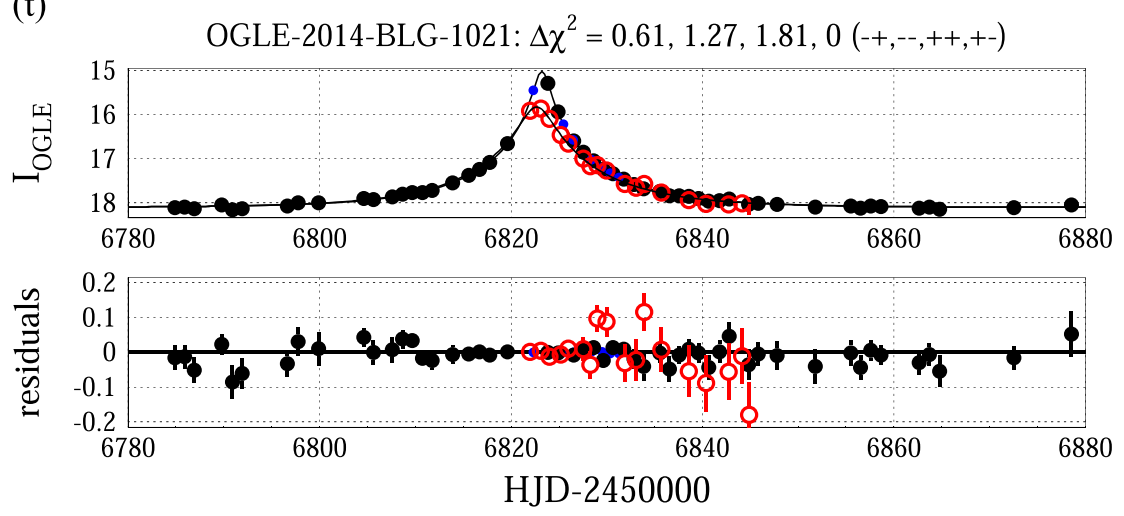

Heliocentric velocity $(\mathrm{km} / \mathrm{s}) \quad$ Geocentric parallax
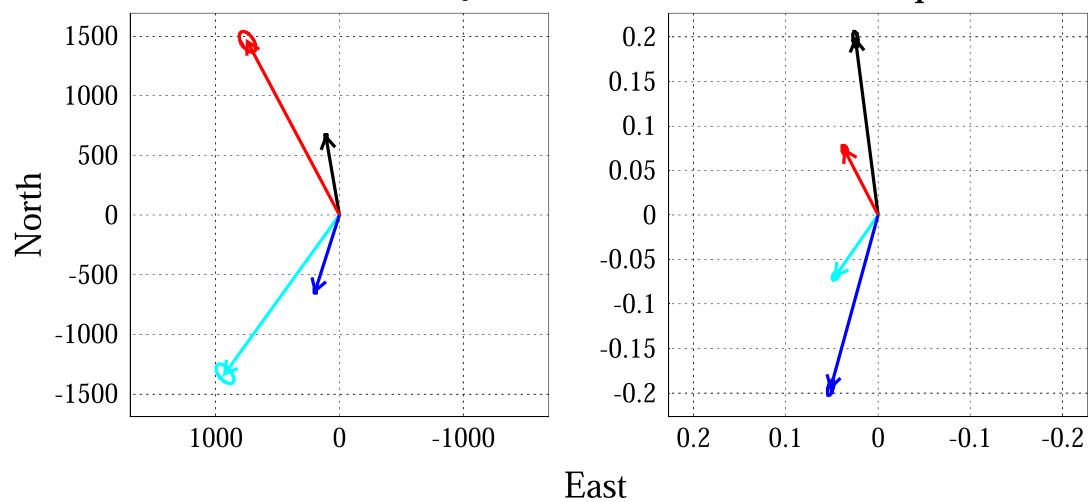

(u)
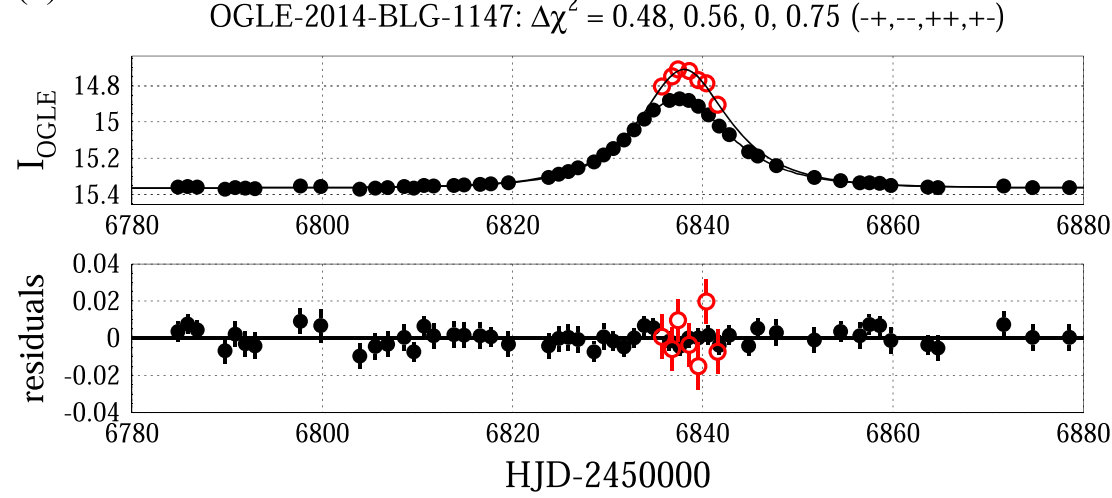

Heliocentric velocity $(\mathrm{km} / \mathrm{s}) \quad$ Geocentric parallax
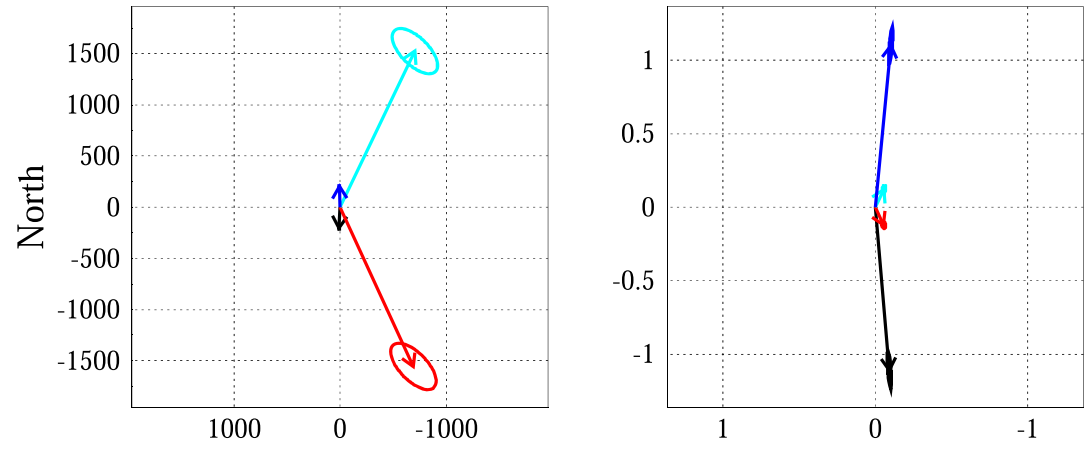

East

Figure 1. (Continued.) 
$\boldsymbol{v}_{\oplus, \perp}(\mathrm{N}, \mathrm{E}) \sim(0,30) \mathrm{km} \mathrm{s}^{-1}$. Hence, $\tilde{\boldsymbol{v}}_{\mathrm{geo}}$ can easily be estimated from these diagrams by eye simply by displacing all vectors by $30 \mathrm{~km} \mathrm{~s}^{-1}$ to the west. (For completeness we note that $\left.\pi_{\mathrm{E}, \text { hel }}=\left(\tilde{\boldsymbol{v}}_{\text {hel }} / \tilde{\nu}_{\text {hel }}\right) \pi_{\mathrm{E}, \text { geo }}.\right)$

These diagrams can be used to judge the relative plausibility of the four solutions. Consider, for example, OGLE-2014BLG-0678. The Spitzer and ground-based light curves are very similar, i.e., similar $t_{0}$ and $u_{0}$. This is what would be expected if $\pi_{\mathrm{E}}$ were very small, and indeed such small $\pi_{\mathrm{E}}$ is apparent for the black $(-+)$ and red (--) solutions in the lower right panel. However, this panel also shows the two $(+ \pm)$ solutions, which have similarly small $\Delta t_{0}$ (so similar $\pi_{\mathrm{E}, E}$ ) to the $(- \pm$ ) solutions, but very different $\Delta u_{0}$ (so $\left.\pi_{\mathrm{E}, N}\right)$. These correspond roughly to $\Delta u_{0} \sim \pm 2 u_{0}$. One of these solutions can clearly be ruled out by its high $\Delta \chi^{2}(++)=24.6$, but the other is only slightly disfavored, $\Delta \chi^{2}(+-)=3.7$.

Nevertheless, following the previously noted argument of James Rich (Section 1.1), both of the $(+ \pm)$ solutions for OGLE-2014-BLG-0678 are highly disfavored. To make the general argument more concrete, we present a "worked example" for this case.

We first note the values, $|\Delta \tau| \sim 0.04,\left|\Delta u_{0,-, \pm}\right| \sim 0.07$, and $u_{0, \oplus} \sim 0.43$ (here $\Delta \tau \equiv \Delta t_{0} / t_{\mathrm{E}, \oplus}$ ), with therefore $|\Delta \tau| \sim\left|\Delta u_{0,-, \pm}\right|$ and additionally $\left|\Delta u_{0,-, \pm}\right| \ll\left|u_{0, \oplus}\right|$. As expected on general grounds, we also find $\left|\Delta u_{0,+, \pm}\right| \sim 0.8 \sim 2\left|u_{0, \oplus}\right|$. We therefore fall within the situation for which the Rich argument applies, $\pi_{\mathrm{E},-} \ll \pi_{\mathrm{E},+}$, and we can conclude that the $\pi_{\mathrm{E},-}$ solution is correct. Indeed, according to Equations (5) and (6), if $\pi_{\mathrm{E},+}$ were correct, with $\epsilon_{0}=0.1$ in this case, the probability of finding such a small ratio would be about $P \sim 0.5 \%$.

More generally, we evaulate the impact of the Rich argument using Equation (6), which was derived in Section 1.1. The argument applies strongly (in the sense that $\pi_{\mathrm{E},+ \pm} \gtrsim 8 \pi_{\mathrm{E},-, \pm}$, i.e., $P<1 / 128)$ to a total of 10 events. Of these 10 , the argument is strongly confirmed by $\Delta \chi^{2}>16$ for three cases (OGLE-2014-BLG-0419, OGLE-2014-BLG-0641, OGLE2014-BLG-0667), and moderately $\left(\Delta \chi^{2}>9\right)$ and marginally $\left(\Delta \chi^{2}>4\right)$ confirmed for one each, OGLE-2014-BLG-0752 and OGLE-2014-BLG-0670, respectively. For four other cases (OGLE-2014-BLG-0678, OGLE-2014-BLG-0866, OGLE2014-BLG-0979, OGLE-2014-BLG-1147) there is no significant information from $\Delta \chi^{2}$. Finally, there is one case (OGLE2014-BLG-0772) for which Rich's argument is marginally contradicted by $\Delta \chi^{2}=7.2$.

The argument applies with moderate strength $\left(2.5 \lesssim \pi_{\mathrm{E},+ \pm} / \pi_{\mathrm{E},-, \pm} \lesssim 8\right)$ to five events (OGLE-2014-BLG0337, OGLE-2014-BLG-0494, OGLE-2014-BLG-0805, OGLE-2014-BLG-0807, OGLE-2014-BLG-0944). There is strong confirmation from $\Delta \chi^{2}$ for the third of these, strong contradiction for the second, and no information from the remaining three.

We conclude that this argument can be reliably applied only to strong cases and should be applied to moderate cases only when significantly confirmed by $\Delta \chi^{2}$. In particular, we note that of the six cases for which the Rich argument was strongly tested by $\Delta \chi^{2}$, the only case for which it was contradicted was OGLE-2014-BLG-0337, i.e., a moderate case with $\pi_{\mathrm{E},+} / \pi_{\mathrm{E},-} \sim 2.5$.

Finally, we note that we have included OGLE-2014-BLG0939 in Figure 1, which was previously analyzed by Yee et al.
(2015), to allow easy comparison with the other isolated-lens events. At the level of analysis of the current paper this would be ranked as a case for which the Rich argument is moderately applicable and is marginally confirmed by $\chi^{2}$. In fact, the source proper-motion measurement carried out by Yee et al. (2015) actually strongly confirms the (--) solution.

Figure 2 illustrates the special case of OGLE-2014-BLG1049. The Earth-based light curve (upper panel) is quite well determined by the combination of OGLE and PLANET SAAO data, which later begin just $7 \mathrm{hr}$ after the high-magnification $\left(u_{0, \oplus}=0.01, A_{\max , \oplus}=100\right)$ peak. By contrast, the Spitzer data, which begin about $13 \mathrm{hr}$ later, leave the peak magnification as seen by Spitzer relatively unconstrained. In particular, $u_{0, \text { Spizer }}$ is consistent with zero, implying that there are a continuum of viable solutions across this "boundary" from $u_{0, \text { Spitzer }}>0$ to $u_{0, \text { Spitzer }}<0$ and hence a merger of the $\Delta u_{0, \pm,+}$ solutions (also of the $\Delta u_{0, \pm,-}$ solutions). The $\pi_{\mathrm{E}, \mathrm{geo}}$ distribution for the $\Delta u_{0, \pm,+}$ solutions is shown in the lower right panel and the corresponding $\tilde{\boldsymbol{v}}_{\text {hel }}$ distribution in the lower left. The $\Delta u_{0, \pm,-}$ solutions (not shown) look extremely similar and have a nearly identical $\chi^{2}$ minimum.

\section{DISTRIBUTION OF LENS DISTANCES}

For each of the 22 isolated-lens events (21 analyzed here plus OGLE-2014-BLG-0939), we calculate the relative likelihood of the lens being at different distances and display our results in Figure 3. As explained below, the abscissa is not the lens distance but rather

$$
D \equiv \frac{\mathrm{kpc}}{\pi_{\mathrm{rel}} / \mathrm{mas}+1 / 8.3},
$$

which has limiting forms

$$
\begin{aligned}
D & \rightarrow D_{L} \quad\left(D_{L} \lesssim D_{S} / 2\right) ; \\
(8.3 \mathrm{kpc}-D) & \rightarrow\left(D_{S}-D_{L}\right) \quad\left(D_{L} \gtrsim D_{S} / 2\right) .
\end{aligned}
$$

The probability distribution is calculated using a restricted set of Bayesian priors, i.e., primarily kinematic priors, combined with the measured values of $\tilde{\boldsymbol{v}}_{\text {hel }}$ and $\pi_{\mathrm{E}}$, as well as discrimination among the four solutions based on $\chi^{2}$ and the Rich argument. That is, there are essentially three factors (in addition to the light-curve-based measurements): phase-space density, $\Delta \chi^{2}$ (displayed above the light curve for each event in Figure 1 and color coded in the bottom panels), and the Rich argument. As discussed in Section 4, the last was applied by suppressing the $(+ \pm)$ solutions, but only for the 10 "strong cases" listed there.

The phase-space density combines the observed value of $\tilde{\boldsymbol{v}}_{\text {hel }}$ with the kinematic priors. It is computed as an integral along the line of sight, with four factors derived from the generic rate equation " $\Gamma=n \sigma v$." The first is a volume element $D_{L}^{2} \Delta D_{L}$. The second is the value of the expected $\tilde{\boldsymbol{v}}$ distribution at the measured value, which we describe below. The third is the "cross section," which is $2 \theta_{\mathrm{E}}=2 \pi_{\mathrm{rel}} / \pi_{\mathrm{E}}$. Since $\pi_{\mathrm{E}}$ is constant along the integral, this factor is effectively $\propto \pi_{\text {rel }}$. The fourth is the "velocity" $\mu=\pi_{\text {rel }} \tilde{v} /$ AU. Again, since $\tilde{v}$ is constant, this term is also $\propto \pi_{\text {rel }}$. Hence, ignoring for the moment the projected-velocity distribution term, the integrand is just $\left(\pi_{\text {rel }} D_{L}\right)^{2} \rightarrow\left(1-D_{L} / D_{S}\right)^{2}$, which falls off fairly slowly in the disk and then drops rapidly in the bulge. 
Table 1

Event Parameters

\begin{tabular}{|c|c|c|c|c|c|c|}
\hline $\begin{array}{l}\text { Event } \\
\text { OGLE-2014-BLG- }\end{array}$ & $\begin{array}{l}\text { R.A. (J2000) } \\
\text { (degree) }\end{array}$ & $\begin{array}{l}\text { Decl. (J2000) } \\
\text { (degree) }\end{array}$ & $\begin{array}{c}\beta_{\mathrm{ec}}(\mathrm{J} 2000) \\
(\text { degree })\end{array}$ & $I-[3.6]$ & $\begin{array}{c}\text { Spitzer } \\
\text { (epochs) }\end{array}$ & Ground-based Data \\
\hline 0099 & 269.607333 & -28.279833 & -4.94030 & $-0.69 \pm 0.06$ & 32 & OGLE, MOA, Wise, RoboNet ${ }^{\mathrm{a}, \mathrm{b}, \mathrm{c}}$, MiNDSTEp $^{\mathrm{d}}$ \\
\hline 0115 & 269.156917 & -28.515750 & -5.17792 & $-0.86 \pm 0.06$ & 22 & OGLE, MOA, Wise, MiNDSTEp ${ }^{d}$ \\
\hline 0419 & 269.629708 & -30.100639 & -6.76105 & $-1.01 \pm 0.07$ & 37 & OGLE, Wise \\
\hline 0494 & 273.191542 & -28.227139 & -4.91827 & $-1.41 \pm 0.15$ & 43 & OGLE, MOA, RoboNet ${ }^{\mathrm{a}, \mathrm{b}, \mathrm{c}}$, MiNDSTEp $^{\mathrm{d}, \mathrm{e}}$ \\
\hline 0589 & 268.380625 & -21.014917 & 2.31661 & $0.54 \pm 0.08$ & 23 & OGLE, RoboNet ${ }^{\mathrm{a}, \mathrm{b}, \mathrm{c}}, \mathrm{MiNDSTEp}^{\mathrm{e}}$ \\
\hline 0670 & 265.542000 & -33.495472 & -10.21366 & $1.16 \pm 0.20$ & 20 & OGLE \\
\hline 0678 & 267.976667 & -31.903389 & -8.57554 & $0.03 \pm 0.10$ & 33 & OGLE \\
\hline 0752 & 270.657333 & -29.594694 & -6.25600 & $-1.13 \pm 0.06$ & 29 & OGLE \\
\hline 0772 & 265.581875 & -23.618861 & -0.34067 & $-0.34 \pm 0.07$ & 26 & OGLE \\
\hline 0805 & 263.152708 & -28.163667 & -4.96693 & $0.34 \pm 0.17$ & 25 & OGLE, PLANET \\
\hline 0807 & 265.186792 & -23.863722 & -0.59693 & $-0.86 \pm 0.09$ & 25 & OGLE, PLANET \\
\hline 1021 & 264.315042 & -29.194722 & -5.95271 & $0.02 \pm 0.10$ & 18 & OGLE, PLANET \\
\hline 1049 & 274.107125 & -31.012333 & -7.72275 & $-2.31 \pm 0.06$ & 19 & OGLE, PLANET \\
\hline 1147 & 261.205875 & -29.600222 & -6.49370 & $-0.35 \pm 0.08$ & 7 & OGLE \\
\hline
\end{tabular}

Note. For the ensemble of the 21 events we report the name, according to the OGLE naming scheme, the coordinates, the instrumental color, $I-$ [3.6], evaluated as discussed in the text, the number of epochs of Spitzer observations, and the ground-based data used for the analysis. The reported instrumental colors are suitable for Spitzer data reduced by a PRF-based analysis; for data reduced by aperture photometry we use an aperture correction factor.

${ }^{\text {a }}$ Siding Spring LCOGT telescope (Australia).

${ }^{\mathrm{b}}$ Sutherland LCOGT telescope (South Africa).

${ }^{c}$ Cerro Tololo LCOGT telescope (Chile).

${ }^{\mathrm{d}}$ Danish telescope, La Silla (Chile).

${ }^{\mathrm{e}}$ Salerno University Telescope (Italy).

All sources were assumed to be in the bulge and to have an isotropic proper-motion dispersion in the bulge frame of $\sigma_{u}=3.0 \mathrm{mas} \mathrm{yr}^{-1}$ (corresponding to $\sim 120 \mathrm{~km} \mathrm{~s}^{-1}$ ) in each direction. Bulge lenses were assumed to have the same propermotion distribution. Disk lenses were assumed to be moving with peculiar motions of dispersions $18 \mathrm{~km} \mathrm{~s}^{-1}$ and $33 \mathrm{~km} \mathrm{~s}^{-1}$ in the vertical and rotation directions relative to a flat rotation curve at $v_{\text {rot }}=240 \mathrm{~km} \mathrm{~s}^{-1}$. The Sun was taken to be moving at 7 and $12 \mathrm{~km} \mathrm{~s}^{-1}$ relative to the same rotation curve.

For disk lenses we simply assumed that the source was at $D_{S}=8.3 \mathrm{kpc}$. Of course, these sources are actually at a range of distances, and the mean distance varies as a function of Galactic longitude due to the tilt of the Galactic bar. However, to first order, our determinations are sensitive only to $\pi_{\text {rel }}$ (rather than to $D_{L}$ and $D_{S}$ separately), so stepping over a discrete set of $D_{S}$ would just yield extremely similar distributions in $\pi_{\text {rel }}$. It is for this reason that we report the quantity " $D$ " in Figure 3, which is a monotonic function of the quantity $\left(\pi_{\text {rel }}\right)$ that we are actually measuring (Equation (14)). The reasons for reporting $D$ rather than $\pi_{\text {rel }}$ itself are twofold. First, $\pi_{\text {rel }}$ is not commonly used as an independent variable, and hence intuition about it is not widespread. This is particularly problematic because many lenses would be bunched up at low $\pi_{\text {rel }}$. More importantly however, the figure as plotted gives direct information about $D_{L}$ for essentially all lenses in the disk (just from the value of $D$ ), and it gives direct information about the distance from the lens to the source for all lenses in or near the bulge from $D_{S}-D_{L} \simeq 8.3 \mathrm{kpc}-D$.
For bulge lenses we conducted an integral over lens distances for each value of " $D$ " by first translating this quantity into $\pi_{\text {rel }}$ and then holding this fixed while allowing $D_{L}$ to vary. We adopted an $r^{-2}$ profile for the bulge, flattened in the vertical direction by a factor 0.6 , and we truncated it at $2 \mathrm{kpc}$. That is, in the above integrals, we weighted by the product of the densities of the lenses and sources, according to the Galactic coordinates of the source.

Since $\pi_{\mathrm{E}}$ is measured, each $\pi_{\mathrm{rel}}$ implies a mass $M=\pi_{\text {rel }} / \kappa \pi_{\mathrm{E}}^{2}$. We truncated the bulge lenses at $M>1.1 M_{\odot}$ and the disk lenses at $M>1.5 M_{\odot}$ due to the paucity of such stars in each population. There may be additional modest constraints on lens mass from (lack of) blended light, but we did not attempt to evaluate these.

As can be seen from Figure 3, the great majority of disk lenses have distance distributions that are relatively compact and characterized by a single peak. This can be understood by inspection of Figure 1. In many cases, one solution is strongly preferred by $\chi^{2}$. OGLE-2014-BLG-0678 provides a good example for which there is no strong preference in $\chi^{2}$ between the two allowed solutions. However, one of the two solutions (black) is closely aligned with the direction of Galactic rotation (roughly $30^{\circ}$ east of north) and so is strongly favored by the kinematic priors. The second (red) solution then contributes almost nothing to the total probability. OGLE-2014-BLG-0670 provides another instructive case. Here the kinematically preferred solution (red) is marginally disfavored by $\Delta \chi^{2}=3.2$. These two factors roughly cancel, but the two solutions predict very similar distance distributions, so the 
Table 2

Event Fit Parameters

\begin{tabular}{|c|c|c|c|c|c|c|c|c|c|c|c|c|}
\hline $\begin{array}{l}\text { Event } \\
\text { OGLE-2014-BLG- }\end{array}$ & $\Delta \chi^{2}$ & $\begin{array}{c}t_{0}-6800 \\
\text { HJD } \\
-2,450,000\end{array}$ & $u_{0}$ & day & $\pi_{\mathrm{E}, \mathrm{N}}$ & $\pi_{\mathrm{E}, \mathrm{E}}$ & $\begin{array}{c}\tilde{v}_{\text {hel, }} \mathrm{N} \\
\left(\mathrm{km} \mathrm{s}^{-1}\right)\end{array}$ & $\begin{array}{c}\tilde{v}_{\text {hel, } \mathrm{E}} \\
\left(\mathrm{km} \mathrm{s}^{-1}\right)\end{array}$ & $I_{\mathrm{OGLE}}$ & $f_{\text {OGLE }^{a}}$ & $\operatorname{mag}_{\text {Spitzer }}{ }^{\mathrm{b}}$ & $f_{\text {Spitzer }}{ }^{a}$ \\
\hline \multirow[t]{8}{*}{0099} & 17.3 & 76.910 & 0.3828 & 116.2 & -0.0823 & 0.2060 & -26.7 & 82.9 & 16.831 & 0.147 & 17.849 & 0.230 \\
\hline & & 0.383 & 0.0067 & 1.3 & 0.0045 & 0.0033 & 1.0 & 1.5 & 0.026 & 0.028 & 0.078 & 0.161 \\
\hline & 0.0 & 76.920 & -0.4075 & 111.1 & 0.1092 & 0.2157 & 27.3 & 78.0 & 16.734 & 0.049 & 17.748 & 0.140 \\
\hline & & -0.407 & 0.0057 & 1.0 & 0.0056 & 0.0036 & 1.0 & 1.4 & 0.021 & 0.021 & 0.071 & 0.137 \\
\hline & 241.5 & 76.949 & 0.2033 & 178.2 & -0.2468 & 0.1594 & -29.6 & 38.4 & 17.728 & 1.618 & 19.042 & 3.534 \\
\hline & & 0.203 & 0.0023 & 1.6 & 0.0025 & 0.0022 & 0.2 & 0.2 & 0.015 & 0.035 & 0.047 & 0.385 \\
\hline & 203.0 & 76.713 & -0.2962 & 127.1 & 0.3698 & 0.1551 & 29.5 & 33.6 & 17.214 & 0.632 & 18.558 & 1.368 \\
\hline & & -0.296 & 0.0029 & 0.9 & 0.0039 & 0.0024 & 0.2 & 0.2 & 0.013 & 0.020 & 0.051 & 0.254 \\
\hline \multirow[t]{8}{*}{0115} & 10.1 & 59.612 & 0.2687 & 105.6 & -0.0777 & 0.1215 & -62.6 & 121.3 & 17.303 & 0.187 & 18.170 & 0.378 \\
\hline & & 0.269 & 0.0036 & 1.0 & 0.0050 & 0.0039 & 2.6 & 4.7 & 0.018 & 0.020 & 0.069 & 0.196 \\
\hline & 0.0 & 59.645 & -0.2867 & 100.5 & 0.0963 & 0.1128 & 73.9 & 113.9 & 17.212 & 0.091 & 18.094 & 0.332 \\
\hline & & -0.287 & 0.0033 & 0.8 & 0.0057 & 0.0040 & 3.1 & 4.6 & 0.016 & 0.016 & 0.063 & 0.179 \\
\hline & 269.6 & 59.257 & 0.2011 & 129.0 & -0.2519 & 0.1232 & -44.4 & 46.7 & 17.693 & 0.701 & 18.818 & 1.704 \\
\hline & & 0.201 & 0.0022 & 1.1 & 0.0034 & 0.0024 & 0.4 & 0.5 & 0.014 & 0.021 & 0.052 & 0.334 \\
\hline & 94.3 & 59.395 & -0.2622 & 102.9 & 0.3416 & 0.0824 & 45.1 & 36.8 & 17.337 & 0.225 & 18.375 & 0.362 \\
\hline & & -0.262 & 0.0027 & 0.8 & 0.0049 & 0.0033 & 0.4 & 0.5 & 0.014 & 0.015 & 0.056 & 0.227 \\
\hline \multirow[t]{8}{*}{0337} & 38.0 & 22.967 & 0.5315 & 45.0 & -0.0790 & 0.1298 & -131.9 & 245.7 & 16.759 & -0.021 & 15.711 & -0.381 \\
\hline & & 0.531 & 0.0077 & 0.4 & 0.0152 & 0.0088 & 17.2 & 24.5 & 0.024 & 0.022 & 0.084 & 0.091 \\
\hline & 19.5 & 23.016 & -0.5465 & 44.4 & 0.3282 & 0.1015 & 108.3 & 62.8 & 16.711 & -0.063 & 16.670 & 1.242 \\
\hline & & -0.547 & 0.0079 & 0.4 & 0.0294 & 0.0102 & 6.4 & 7.6 & 0.024 & 0.021 & 0.157 & 0.439 \\
\hline & 1611.6 & 22.472 & 0.4691 & 48.8 & -0.6151 & 0.0715 & -57.1 & 35.8 & 16.959 & 0.178 & 18.399 & 14.687 \\
\hline & & 0.469 & 0.0056 & 0.4 & 0.0091 & 0.0039 & 0.5 & 0.4 & 0.019 & 0.021 & 0.078 & 1.295 \\
\hline & 0.0 & 23.022 & -0.6558 & 38.9 & 0.9844 & -0.2310 & 42.7 & 19.1 & 16.385 & -0.306 & 16.616 & 1.036 \\
\hline & & -0.656 & 0.0121 & 0.5 & 0.0295 & 0.0208 & 1.1 & 0.4 & 0.034 & 0.022 & 0.161 & 0.427 \\
\hline \multirow[t]{8}{*}{0419} & 0.6 & 22.886 & 0.2401 & 48.6 & -0.0253 & -0.0334 & -513.3 & -648.8 & 18.202 & 0.328 & 19.391 & 0.430 \\
\hline & & 0.240 & 0.0048 & 0.7 & 0.0071 & 0.0033 & 86.6 & 132.2 & 0.026 & 0.031 & 0.058 & 0.136 \\
\hline & 0.0 & 22.896 & -0.2414 & 48.4 & 0.0185 & -0.0417 & 318.3 & -687.2 & 18.195 & 0.320 & 19.400 & 0.441 \\
\hline & & -0.241 & 0.0049 & 0.7 & 0.0077 & 0.0032 & 96.5 & 104.5 & 0.026 & 0.031 & 0.058 & 0.136 \\
\hline & 94.4 & 22.763 & 0.2345 & 49.4 & -0.4832 & -0.0630 & -70.9 & 19.8 & 18.230 & 0.363 & 19.447 & 0.578 \\
\hline & & 0.235 & 0.0047 & 0.7 & 0.0114 & 0.0040 & 1.1 & 0.4 & 0.026 & 0.032 & 0.058 & 0.144 \\
\hline & 23.4 & 22.954 & -0.2549 & 46.5 & 0.4700 & -0.1851 & 68.9 & 2.1 & 18.122 & 0.234 & 19.419 & 0.325 \\
\hline & & -0.255 & 0.0052 & 0.6 & 0.0107 & 0.0058 & 1.0 & 0.4 & 0.027 & 0.030 & 0.059 & 0.141 \\
\hline \multirow[t]{8}{*}{0494} & 5.9 & 17.310 & 0.1540 & 33.2 & 0.0381 & 0.0953 & 189.5 & 499.7 & 14.493 & -0.004 & 16.138 & 0.212 \\
\hline & & 0.154 & 0.0005 & 0.1 & 0.0027 & 0.0029 & 5.4 & 18.8 & 0.004 & 0.004 & 0.016 & 0.021 \\
\hline & 0.0 & 17.306 & -0.1539 & 33.3 & -0.0048 & 0.1074 & -20.4 & 511.8 & 14.493 & -0.003 & 16.129 & 0.204 \\
\hline & & -0.154 & 0.0005 & 0.1 & 0.0018 & 0.0037 & 7.3 & 17.0 & 0.004 & 0.004 & 0.017 & 0.021 \\
\hline & 160.5 & 17.278 & 0.1547 & 33.2 & -0.4448 & 0.1101 & -109.0 & 55.6 & 14.488 & -0.008 & 16.131 & 0.216 \\
\hline & & 0.155 & 0.0005 & 0.1 & 0.0028 & 0.0029 & 0.8 & 0.4 & 0.004 & 0.004 & 0.016 & 0.021 \\
\hline & 233.9 & 17.340 & -0.1552 & 33.1 & 0.4445 & -0.0276 & 118.5 & 21.0 & 14.482 & -0.014 & 16.199 & 0.268 \\
\hline & & -0.155 & 0.0005 & 0.1 & 0.0032 & 0.0022 & 0.7 & 0.7 & 0.004 & 0.004 & 0.016 & 0.022 \\
\hline \multirow[t]{8}{*}{0589} & 1.9 & 7.622 & 0.0518 & 33.9 & 0.3727 & 0.1983 & 106.1 & 84.3 & 16.942 & -0.052 & 16.703 & 0.118 \\
\hline & & 0.052 & 0.0006 & 0.3 & 0.0286 & 0.0333 & 8.0 & 9.7 & 0.012 & 0.010 & 0.070 & 0.075 \\
\hline & 1.0 & 7.624 & -0.0516 & 34.0 & -0.3700 & 0.2040 & -106.3 & 85.7 & 16.946 & -0.049 & 16.657 & 0.071 \\
\hline & & -0.052 & 0.0006 & 0.3 & 0.0293 & 0.0337 & 8.3 & 9.6 & 0.012 & 0.010 & 0.071 & 0.073 \\
\hline & 0.0 & 7.624 & 0.0517 & 34.1 & -0.5368 & 0.1728 & -86.6 & 55.2 & 16.945 & -0.049 & 16.639 & 0.051 \\
\hline & & 0.052 & 0.0006 & 0.3 & 0.0303 & 0.0350 & 4.4 & 5.9 & 0.012 & 0.010 & 0.072 & 0.072 \\
\hline & 0.7 & 7.622 & -0.0519 & 33.9 & 0.5544 & 0.1583 & 84.4 & 51.8 & 16.939 & -0.054 & 16.701 & 0.115 \\
\hline & & -0.052 & 0.0006 & 0.3 & 0.0277 & 0.0335 & 4.0 & 5.3 & 0.012 & 0.010 & 0.071 & 0.075 \\
\hline \multirow[t]{8}{*}{0641} & 0.0 & 46.959 & 0.5398 & 38.6 & -0.0182 & 0.0333 & -568.5 & 1062.6 & 16.876 & -0.071 & 18.015 & 0.311 \\
\hline & & 0.540 & 0.0162 & 0.7 & 0.0106 & 0.0100 & 218.1 & 398.3 & 0.050 & 0.043 & 0.080 & 0.110 \\
\hline & 0.9 & 46.974 & -0.5411 & 38.6 & 0.0202 & 0.0327 & 610.3 & 1021.2 & 16.871 & -0.075 & 17.992 & 0.277 \\
\hline & & -0.541 & 0.0163 & 0.7 & 0.0121 & 0.0103 & 246.1 & 418.0 & 0.050 & 0.043 & 0.077 & 0.104 \\
\hline & 318.9 & 46.465 & 0.4434 & 42.9 & -0.6919 & 0.0509 & -60.2 & 32.1 & 17.197 & 0.251 & 18.614 & 1.451 \\
\hline & & 0.443 & 0.0116 & 0.7 & 0.0194 & 0.0069 & 0.8 & 0.7 & 0.041 & 0.047 & 0.064 & 0.165 \\
\hline & 23.7 & 46.971 & -0.5893 & 35.1 & 0.8950 & -0.1728 & 51.0 & 17.5 & 16.722 & -0.193 & 17.882 & 0.028 \\
\hline & & -0.589 & 0.0188 & 0.7 & 0.0286 & 0.0140 & 0.8 & 0.5 & 0.055 & 0.041 & 0.082 & 0.097 \\
\hline \multirow[t]{6}{*}{0667} & 0.0 & 35.115 & 0.4672 & 32.3 & 0.1128 & 0.0386 & 426.1 & 174.8 & 16.161 & 0.022 & 17.280 & 0.224 \\
\hline & & 0.467 & 0.0096 & 0.4 & 0.0135 & 0.0061 & 31.2 & 46.1 & 0.032 & 0.030 & 0.075 & 0.106 \\
\hline & 8.9 & 35.093 & -0.4656 & 32.4 & -0.1021 & 0.0588 & -392.9 & 255.8 & 16.166 & 0.027 & 17.311 & 0.275 \\
\hline & & -0.466 & 0.0095 & 0.4 & 0.0146 & 0.0050 & 23.9 & 54.2 & 0.032 & 0.031 & 0.075 & 0.109 \\
\hline & 285.3 & 34.818 & 0.4739 & 31.7 & -0.9800 & -0.0669 & -54.9 & 25.5 & 16.137 & 0.000 & 17.485 & 0.553 \\
\hline & & 0.474 & 0.0102 & 0.4 & 0.0234 & 0.0086 & 0.8 & 0.4 & 0.034 & 0.031 & 0.076 & 0.132 \\
\hline
\end{tabular}


Table 2

(Continued)

\begin{tabular}{|c|c|c|c|c|c|c|c|c|c|c|c|c|}
\hline $\begin{array}{l}\text { Event } \\
\text { OGLE-2014-BLG- }\end{array}$ & $\Delta \chi^{2}$ & $\begin{array}{c}t_{0}-6800 \\
\text { HJD } \\
-2,450,000\end{array}$ & $u_{0}$ & day & $\pi_{\mathrm{E}, \mathrm{N}}$ & $\pi_{\mathrm{E}, \mathrm{E}}$ & $\begin{array}{c}\tilde{v}_{\text {hel, } \mathrm{N}} \\
\left(\mathrm{km} \mathrm{s}^{-1}\right)\end{array}$ & $\begin{array}{c}\tilde{v}_{\text {hel, E }} \\
\left(\mathrm{km} \mathrm{s}^{-1}\right)\end{array}$ & $I_{\mathrm{OGLE}}$ & $f_{\mathrm{OGLE}}{ }^{\mathrm{a}}$ & $\operatorname{mag}_{\text {Spitzer }} \mathrm{b}$ & $f_{\text {Spitzer }}{ }^{\mathrm{a}}$ \\
\hline \multirow{8}{*}{0670} & 59.8 & 34.999 & -0.5000 & 30.6 & 1.0009 & -0.2452 & 53.8 & 16.2 & 16.050 & -0.078 & 17.261 & 0.144 \\
\hline & & -0.500 & 0.0111 & 0.4 & 0.0231 & 0.0122 & 0.8 & 0.3 & 0.036 & 0.031 & 0.079 & 0.109 \\
\hline & 3.2 & 15.144 & 0.7697 & 25.6 & 0.0557 & 0.0928 & 321.4 & 564.3 & 16.049 & 0.084 & 14.946 & 0.539 \\
\hline & & 0.770 & 0.0934 & 2.0 & 0.0697 & 0.0300 & 238.6 & 360.8 & 0.239 & 0.239 & 0.296 & 0.425 \\
\hline & 0.0 & 15.105 & -0.7478 & 26.2 & -0.1053 & 0.1485 & -210.1 & 325.2 & 16.106 & 0.142 & 14.777 & 0.310 \\
\hline & & -0.748 & 0.0890 & 2.0 & 0.0809 & 0.0436 & 82.1 & 162.6 & 0.232 & 0.243 & 0.291 & 0.355 \\
\hline & 5.2 & 14.597 & 0.7250 & 27.0 & -1.7211 & -0.2294 & -36.5 & 23.9 & 16.162 & 0.203 & 14.978 & 0.579 \\
\hline & & 0.725 & 0.0881 & 2.1 & 0.2054 & 0.0596 & 2.1 & 0.7 & 0.233 & 0.258 & 0.289 & 0.428 \\
\hline \multirow{5}{*}{0678} & 32.7 & 15.481 & -0.9690 & 21.9 & 1.8265 & -0.9361 & 34.4 & 11.3 & 15.560 & -0.309 & 14.635 & 0.074 \\
\hline & & -0.969 & 0.1709 & 2.5 & 0.2888 & 0.2070 & 2.5 & 0.6 & 0.391 & 0.248 & 0.437 & 0.453 \\
\hline & 0.5 & 22.017 & 0.4260 & 30.3 & -0.0556 & 0.0660 & -426.8 & 536.0 & 17.467 & 0.267 & 17.381 & 0.309 \\
\hline & & 0.426 & 0.0212 & 1.0 & 0.0199 & 0.0105 & 76.4 & 151.7 & 0.076 & 0.088 & 0.107 & 0.156 \\
\hline & 0.0 & 22.034 & -0.4284 & 30.2 & 0.0810 & 0.0532 & 494.6 & 353.8 & 17.459 & 0.257 & 17.389 & 0.313 \\
\hline \multirow{11}{*}{0752} & & -0.428 & 0.0214 & 1.0 & 0.0212 & 0.0117 & 71.5 & 125.6 & 0.076 & 0.088 & 0.108 & 0.156 \\
\hline & 24.6 & 21.863 & 0.4149 & 30.8 & -0.8523 & 0.0245 & -65.7 & 31.0 & 17.506 & 0.312 & 17.413 & 0.383 \\
\hline & & 0.415 & 0.0207 & 1.0 & 0.0455 & 0.0111 & 1.9 & 0.9 & 0.075 & 0.091 & 0.107 & 0.162 \\
\hline & 3.7 & 22.111 & -0.4541 & 29.0 & 0.8474 & -0.2429 & 65.2 & 10.5 & 17.367 & 0.155 & 17.395 & 0.215 \\
\hline & & -0.454 & 0.0235 & 1.0 & 0.0446 & 0.0193 & 1.8 & 0.7 & 0.081 & 0.086 & 0.111 & 0.160 \\
\hline & 0.8 & 39.353 & 0.6781 & 39.7 & 0.0669 & 0.0381 & 491.7 & 309.0 & 16.682 & 0.609 & 17.816 & 0.409 \\
\hline & & 0.678 & 0.0500 & 1.9 & 0.0165 & 0.0106 & 56.8 & 131.1 & 0.138 & 0.204 & 0.154 & 0.209 \\
\hline & 0.0 & 39.311 & -0.6611 & 40.5 & -0.0664 & 0.0537 & -389.6 & 343.6 & 16.730 & 0.681 & 17.857 & 0.474 \\
\hline & & -0.661 & 0.0482 & 1.9 & 0.0189 & 0.0107 & 53.7 & 114.8 & 0.134 & 0.208 & 0.149 & 0.212 \\
\hline & 137.1 & 38.384 & 0.4633 & 49.7 & -0.8725 & -0.0944 & -39.7 & 24.8 & 17.340 & 1.949 & 18.565 & 1.878 \\
\hline & & 0.463 & 0.0261 & 1.9 & 0.0469 & 0.0132 & 0.8 & 0.4 & 0.090 & 0.244 & 0.107 & 0.307 \\
\hline \multirow{5}{*}{0772} & 11.8 & 38.951 & -0.7303 & 36.1 & 1.1933 & -0.3988 & 35.8 & 16.9 & 16.537 & 0.408 & 17.682 & 0.079 \\
\hline & & -0.730 & 0.0564 & 1.9 & 0.0831 & 0.0384 & 0.9 & 0.2 & 0.149 & 0.193 & 0.163 & 0.183 \\
\hline & 7.2 & 17.428 & 0.4645 & 26.9 & -0.0308 & 0.0161 & -1643.0 & 886.4 & 17.066 & 0.727 & 17.353 & 0.417 \\
\hline & & 0.464 & 0.0308 & 1.1 & 0.0124 & 0.0046 & 404.1 & 528.5 & 0.104 & 0.166 & 0.089 & 0.112 \\
\hline & 7.2 & 17.429 & -0.4644 & 26.9 & 0.0304 & 0.0168 & 1618.8 & 923.9 & 17.066 & 0.727 & 17.353 & 0.417 \\
\hline \multirow{11}{*}{0805} & & -0.464 & 0.0308 & 1.1 & 0.0125 & 0.0046 & 394.7 & 536.3 & 0.104 & 0.166 & 0.089 & 0.112 \\
\hline & 0.0 & 17.403 & 0.4684 & 26.8 & -0.9883 & -0.2107 & -63.3 & 15.7 & 17.051 & 0.703 & 17.403 & 0.462 \\
\hline & & 0.468 & 0.0308 & 1.1 & 0.0583 & 0.0150 & 1.3 & 0.3 & 0.104 & 0.162 & 0.084 & 0.111 \\
\hline & 1.6 & 17.415 & -0.4687 & 26.8 & 0.9903 & -0.1883 & 62.1 & 17.0 & 17.050 & 0.701 & 17.412 & 0.472 \\
\hline & & -0.469 & 0.0308 & 1.1 & 0.0583 & 0.0136 & 1.3 & 0.2 & 0.104 & 0.162 & 0.084 & 0.111 \\
\hline & 0.1 & 39.907 & 0.1794 & 55.8 & -0.0586 & 0.0289 & -428.4 & 238.4 & 18.593 & 0.067 & 18.583 & 2.236 \\
\hline & & 0.179 & 0.0092 & 2.1 & 0.0054 & 0.0023 & 24.1 & 21.9 & 0.064 & 0.062 & 0.069 & 0.290 \\
\hline & 0.0 & 39.919 & -0.1808 & 55.4 & 0.0609 & 0.0288 & 417.1 & 226.3 & 18.582 & 0.056 & 18.588 & 2.241 \\
\hline & & -0.181 & 0.0093 & 2.1 & 0.0056 & 0.0023 & 23.5 & 21.0 & 0.064 & 0.062 & 0.069 & 0.291 \\
\hline & 1.6 & 39.865 & 0.1759 & 56.4 & -0.2461 & 0.0157 & -126.2 & 36.1 & 18.618 & 0.092 & 18.593 & 2.283 \\
\hline & & 0.176 & 0.0091 & 2.1 & 0.0120 & 0.0021 & 2.0 & 1.2 & 0.064 & 0.063 & 0.069 & 0.291 \\
\hline \multirow{5}{*}{0807} & 0.1 & 39.918 & -0.1823 & 54.8 & 0.2497 & 0.0120 & 124.2 & 34.2 & 18.572 & 0.047 & 18.614 & 2.299 \\
\hline & & -0.182 & 0.0094 & 2.1 & 0.0121 & 0.0021 & 2.0 & 1.1 & 0.064 & 0.061 & 0.068 & 0.295 \\
\hline & 0.0 & 30.101 & 0.0630 & 182.8 & -0.0153 & -0.0385 & -85.4 & -183.3 & 20.963 & 5.784 & 21.898 & 6.906 \\
\hline & & 0.063 & 0.0170 & 43.7 & 0.0051 & 0.0096 & 14.8 & 11.8 & 0.308 & 1.900 & 0.315 & 2.765 \\
\hline & 0.1 & 30.103 & -0.0632 & 182.2 & 0.0164 & -0.0383 & 88.7 & -180.5 & 20.958 & 5.755 & 21.894 & 6.859 \\
\hline \multirow{11}{*}{0866} & & -0.063 & 0.0171 & 43.4 & 0.0053 & 0.0095 & 14.7 & 11.8 & 0.306 & 1.884 & 0.314 & 2.745 \\
\hline & 0.6 & 30.059 & 0.0626 & 183.2 & -0.0921 & -0.0483 & -81.5 & -13.0 & 20.969 & 5.831 & 21.910 & 6.824 \\
\hline & & 0.063 & 0.0170 & 44.0 & 0.0248 & 0.0124 & 3.0 & 2.5 & 0.309 & 1.926 & 0.318 & 2.790 \\
\hline & 0.8 & 30.069 & -0.0636 & 180.5 & 0.0947 & -0.0464 & 80.7 & -10.8 & 20.951 & 5.717 & 21.890 & 6.612 \\
\hline & & -0.064 & 0.0172 & 43.0 & 0.0253 & 0.0118 & 3.0 & 2.4 & 0.308 & 1.881 & 0.312 & 2.697 \\
\hline & 2.7 & 14.116 & 0.4062 & 17.3 & 0.1300 & 0.0254 & 739.8 & 172.9 & 17.523 & 0.211 & 17.813 & 0.450 \\
\hline & & 0.406 & 0.0383 & 1.0 & 0.0284 & 0.0226 & 176.7 & 84.4 & 0.142 & 0.158 & 0.172 & 0.228 \\
\hline & 2.7 & 14.115 & -0.4062 & 17.3 & -0.1298 & 0.0259 & -740.2 & 175.9 & 17.523 & 0.211 & 17.814 & 0.450 \\
\hline & & -0.406 & 0.0383 & 1.0 & 0.0283 & 0.0227 & 177.0 & 84.1 & 0.142 & 0.158 & 0.172 & 0.228 \\
\hline & 0.1 & 14.119 & 0.4183 & 17.1 & -1.1858 & -0.1739 & -84.0 & 16.2 & 17.478 & 0.162 & 17.801 & 0.429 \\
\hline & & 0.418 & 0.0405 & 1.0 & 0.1158 & 0.0220 & 3.4 & 1.5 & 0.147 & 0.157 & 0.175 & 0.229 \\
\hline \multirow{6}{*}{0874} & 0.0 & 14.121 & -0.4186 & 17.1 & 1.1861 & -0.1788 & 83.2 & 15.9 & 17.477 & 0.161 & 17.799 & 0.427 \\
\hline & & -0.419 & 0.0406 & 1.0 & 0.1159 & 0.0223 & 3.4 & 1.5 & 0.147 & 0.157 & 0.175 & 0.229 \\
\hline & 19.7 & 45.665 & 0.1852 & 25.5 & -0.1028 & 0.0212 & -635.1 & 159.2 & 15.908 & 0.023 & 17.350 & 1.387 \\
\hline & & 0.185 & 0.0007 & 0.1 & 0.0052 & 0.0040 & 29.9 & 26.9 & 0.005 & 0.004 & 0.059 & 0.247 \\
\hline & 3.1 & 45.668 & -0.1855 & 25.4 & 0.1074 & 0.0092 & 629.0 & 82.1 & 15.906 & 0.021 & 17.377 & 1.456 \\
\hline & & -0.186 & 0.0007 & 0.1 & 0.0049 & 0.0039 & 27.9 & 23.9 & 0.005 & 0.004 & 0.059 & 0.252 \\
\hline
\end{tabular}


Table 2

(Continued)

\begin{tabular}{|c|c|c|c|c|c|c|c|c|c|c|c|c|}
\hline $\begin{array}{l}\text { Event } \\
\text { OGLE-2014-BLG- }\end{array}$ & $\Delta \chi^{2}$ & $\begin{array}{c}t_{0}-6800 \\
\text { HJD } \\
-2,450,000\end{array}$ & $u_{0}$ & day & $\pi_{\mathrm{E}, \mathrm{N}}$ & $\pi_{\mathrm{E}, \mathrm{E}}$ & $\begin{array}{c}\tilde{v}_{\text {hel, }} \mathrm{N} \\
\left(\mathrm{km} \mathrm{s}^{-1}\right)\end{array}$ & $\begin{array}{c}\tilde{v}_{\text {hel, }} \mathrm{E} \\
\left(\mathrm{km} \mathrm{s}^{-1}\right)\end{array}$ & $I_{\mathrm{OGLE}}$ & $f_{\text {OGLE }}{ }^{\mathrm{a}}$ & $\operatorname{mag}_{\text {Spitzer }} \mathrm{b}$ & $f_{\text {Spitzer }}{ }^{\mathrm{a}}$ \\
\hline \multirow{9}{*}{0944} & 36.4 & 45.662 & 0.1850 & 25.5 & -0.2097 & 0.0225 & -321.0 & 62.7 & 15.910 & 0.024 & 17.382 & 1.494 \\
\hline & 0.0 & 45.669 & -0.1857 & 25.4 & 0.2132 & -0.0002 & 319.3 & 28.0 & 15.905 & 0.020 & 17.374 & 1.434 \\
\hline & & -0.186 & 0.0007 & 0.1 & 0.0051 & 0.0038 & 7.5 & 5.7 & 0.005 & 0.004 & 0.059 & 0.252 \\
\hline & 0.6 & 12.751 & 0.2742 & 9.9 & 0.0801 & -0.1700 & 397.2 & -815.4 & 15.668 & -0.085 & 15.648 & 0.099 \\
\hline & & -0.274 & 0.0077 & 0.2 & 0.0103 & 0.0129 & 72.7 & 26.1 & 0.036 & 0.031 & 0.064 & 0.065 \\
\hline & 1.7 & 12.749 & 0.2741 & 9.9 & -0.7638 & -0.1578 & -220.9 & -16.7 & 15.669 & -0.084 & 15.605 & 0.056 \\
\hline & & 0.274 & 0.0077 & 0.2 & 0.0245 & 0.0122 & 2.7 & 4.6 & 0.036 & 0.031 & 0.065 & 0.064 \\
\hline & 0.0 & 12.752 & -0.2744 & 9.9 & 0.7075 & -0.2602 & 217.6 & -51.5 & 15.667 & -0.085 & 15.700 & 0.151 \\
\hline & & -0.274 & 0.0077 & 0.2 & 0.0231 & 0.0105 & 2.1 & 4.7 & 0.037 & 0.031 & 0.061 & 0.066 \\
\hline \multirow[t]{4}{*}{0979} & 0.0 & 13.737 & 0.1064 & 8.9 & -0.0076 & -0.0330 & -1291.2 & -5550.2 & 17.460 & 0.108 & 18.925 & -0.162 \\
\hline & & 0.106 & 0.0044 & 0.3 & 0.0116 & 0.0170 & 10.8 & 45.3 & 0.049 & 0.050 & 0.088 & 0.076 \\
\hline & 0.2 & 13.738 & -0.1065 & 8.9 & 0.2440 & -0.1105 & 660.7 & -270.1 & 17.460 & 0.107 & 18.948 & -0.150 \\
\hline & & -0.106 & 0.0044 & 0.3 & 0.0113 & 0.0162 & 27.2 & 37.6 & 0.049 & 0.050 & 0.087 & 0.077 \\
\hline \multirow[t]{8}{*}{1021} & 0.6 & 23.214 & 0.0604 & 13.2 & 0.0729 & 0.0359 & 1451.7 & 744.3 & 18.072 & -0.028 & 17.998 & 2.126 \\
\hline & & 0.060 & 0.0027 & 0.4 & 0.0051 & 0.0031 & 86.9 & 66.1 & 0.047 & 0.042 & 0.056 & 0.172 \\
\hline & 1.3 & 23.214 & -0.0604 & 13.2 & -0.0680 & 0.0458 & -1331.6 & 926.6 & 18.072 & -0.028 & 17.992 & 2.111 \\
\hline & & -0.060 & 0.0027 & 0.4 & 0.0049 & 0.0035 & 81.5 & 72.2 & 0.047 & 0.042 & 0.057 & 0.172 \\
\hline & 1.8 & 23.214 & 0.0604 & 13.2 & -0.1940 & 0.0513 & -635.1 & 196.8 & 18.073 & -0.028 & 17.987 & 2.098 \\
\hline & & 0.060 & 0.0027 & 0.4 & 0.0088 & 0.0035 & 16.9 & 9.0 & 0.047 & 0.042 & 0.057 & 0.172 \\
\hline & 0.0 & 23.214 & -0.0604 & 13.2 & 0.1973 & 0.0243 & 656.1 & 110.4 & 18.072 & -0.028 & 18.004 & 2.141 \\
\hline & & -0.060 & 0.0027 & 0.4 & 0.0089 & 0.0029 & 16.9 & 8.9 & 0.047 & 0.042 & 0.056 & 0.173 \\
\hline \multirow[t]{2}{*}{1147} & 0.5 & 37.488 & 0.7191 & 7.7 & -0.1197 & -0.0554 & -1554.7 & -689.8 & 15.487 & 0.121 & 15.834 & 0.756 \\
\hline & & 0.719 & 0.0854 & 0.6 & 0.0254 & 0.0112 & 225.8 & 213.7 & 0.227 & 0.234 & 0.240 & 0.415 \\
\hline
\end{tabular}

Note. Fit parameters for the ensemble of 20 out the 21 events discussed in the text. For the analysis of OGLE-2014-BLG-1049 we refer to the text and Figure 2. For each event we report the four solutions in the order,,,-+--+++- . The light curves and the ellipses for each solution for the heliocentric velocity and the parallax are show in Figure 1.

${ }_{\mathrm{b}}^{\mathrm{a}} \mathrm{f}$ indicates the ratio of blend to source flux.

${ }^{\mathrm{b}}$ Instrumental magnitude.

combined probability distribution function is only slightly broadened by the ambiguity. In fact, of all the lenses in the sample, there are only two that are double-peaked: OGLE2014-BLG-0944 and OGLE-2014-BLG-1021. In both cases, the $(- \pm)$ solutions correspond to bulge lenses while the $(+ \pm)$ solutions correspond to disk lenses. And in both cases, $\chi^{2}$ does very little to discriminate between possible solutions. Hence, we treat the bulge and disk solutions as equally likely in each case. The resulting double-peaked probability distributions are shown as bold dashed curves in Figure 3. Of the six other bulge-lens events, one has somewhat double-peaked features due to slightly different $\tilde{v}$ and the fact that the direction of $\tilde{\boldsymbol{v}}$ does not differentiate between solutions for bulge lenses (because of the assumed isotropy of proper motions).

Note that the Galactic model used for the distance measurements is simplified in a number of respects. First, there is no weighting by an assumed lens mass function, which is equivalent to assuming a flat prior in log mass. Second, for the disk lenses, there is no weighting by stellar density, which is equivalent to assuming that the declining density with distance from the plane due to the vertical scale height exactly cancels the increasing density as one approaches the Galactic center due to the radial scale length. And of course, we do not attempt to model even finer details, such as varying velocity dispersion, changing scale heights, etc.

We do not develop more sophisticated models for three reasons. First, we wish to demonstrate the power of kinematic priors (combined with $\tilde{\boldsymbol{v}}$ measurements) alone to constrain the distances to individual lenses. This point has been made before theoretically (Han \& Gould 1995), but has never been demonstrated practically.

Second, the distance measurements individually, and especially cumulatively, are robust against modest changes in assumptions. For example, if $v_{\text {rot }}$ is changed from 240 to 

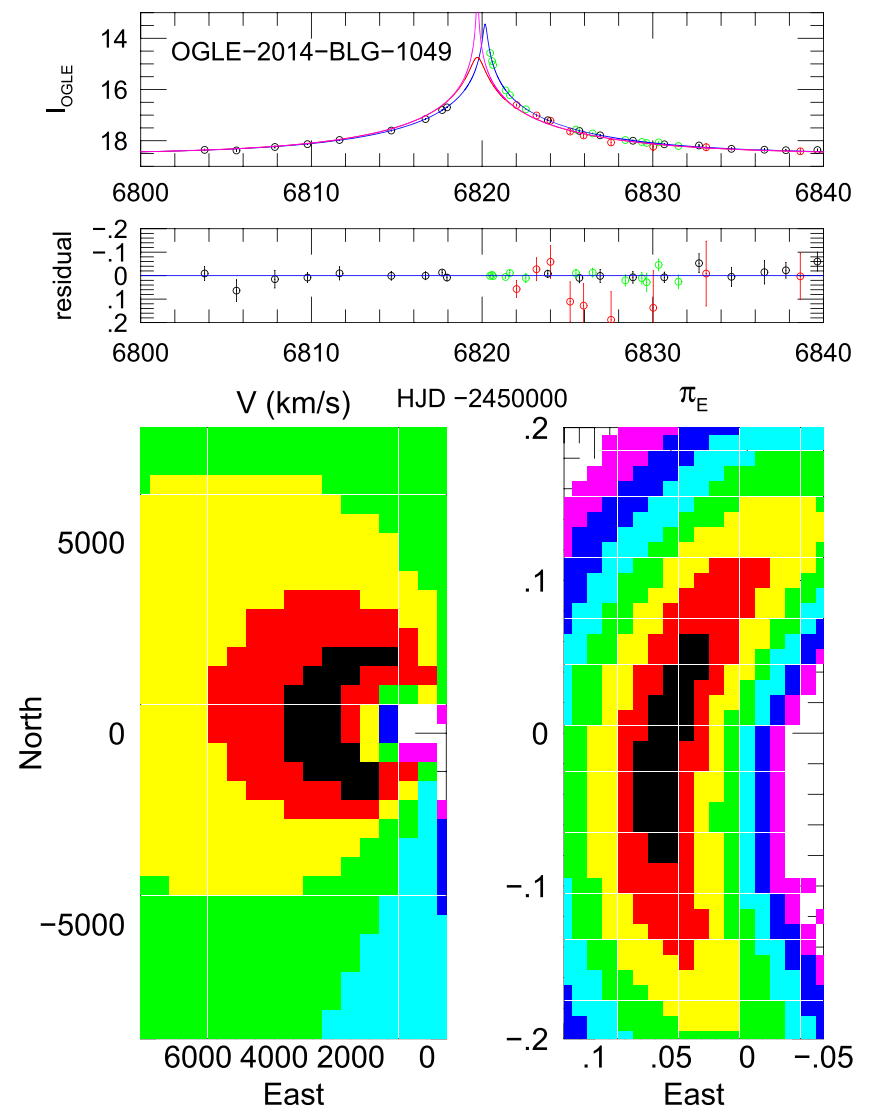

Figure 2. Top panel: OGLE-2014-BLG-1049 light curves for OGLE (black), PLANET SAAO (green), and Spitzer (red) data. Ground-based model (blue) is well defined, but many models are consistent with Spitzer data (e.g., red and magenta curves). Middle panel: residuals. Lower panel: $\Delta \chi^{2}$ offsets $(1,4,9$, ...) from minimum for geocentric parallax $\pi_{\mathrm{E}, \mathrm{geo}}($ right) and heliocentric proper motion $\tilde{v}_{\text {hel }}$ (left) for $u_{0, \oplus}>0$ solution (merger of $\Delta u_{0, \pm,+}$ solutions). The $\Delta u_{0, \pm,-}$ solutions (not shown) are extremely similar. Because $u_{0, \text { Spitzer }}$ is more poorly defined than $t_{0, \text { Spitzer }}$ (top panel), $\Delta u_{0}$ is relatively uncertain, which translates directly into uncertainty in $\pi_{\mathrm{E} \text {,north }}$ because the Earth-Spitzer axis is almost due east-west.

$220 \mathrm{~km} \mathrm{~s}^{-1}$, then the resulting version of Figure 3 is indistinguishable by eye from the current one. As another example, we have recomputed the distance distributions in Figure 3 using more realistic mass priors $d N / d \ln M \propto M^{-x}$, with $x=0.3(1.3)$ for $M>(<) 0.5 M_{\odot}$ for the disk and $x=1$ $(0.3)$ for $M>(<) 0.7 M_{\odot}$ for the bulge. We plot the resulting cumulative distributions in Figure 3 with (solid) and without (bold) mass-function priors and note that they hardly differ. The reason for this is that over the regions of parameter space permitted by the kinematic priors, the mass priors generally do not vary very much.

Third, the proper context to study the impact of model variations is within a determination of the Galactic distribution of planets. As we discuss in Section 6 immediately below, such a measurement will require additional data.

\section{PATHWAY TO GALACTIC DISTRIBUTION OF PLANETS}

Figure 3 shows the cumulative distribution of $D$ (monotonic function of $\pi_{\text {rel }}$ ), constructed by adding together all the lens probability distributions and normalizing to unity. The position of the one planet in the Spitzer sample (OGLE-2014-BLG0124; Udalski et al. 2015) is also shown. Of course, nothing

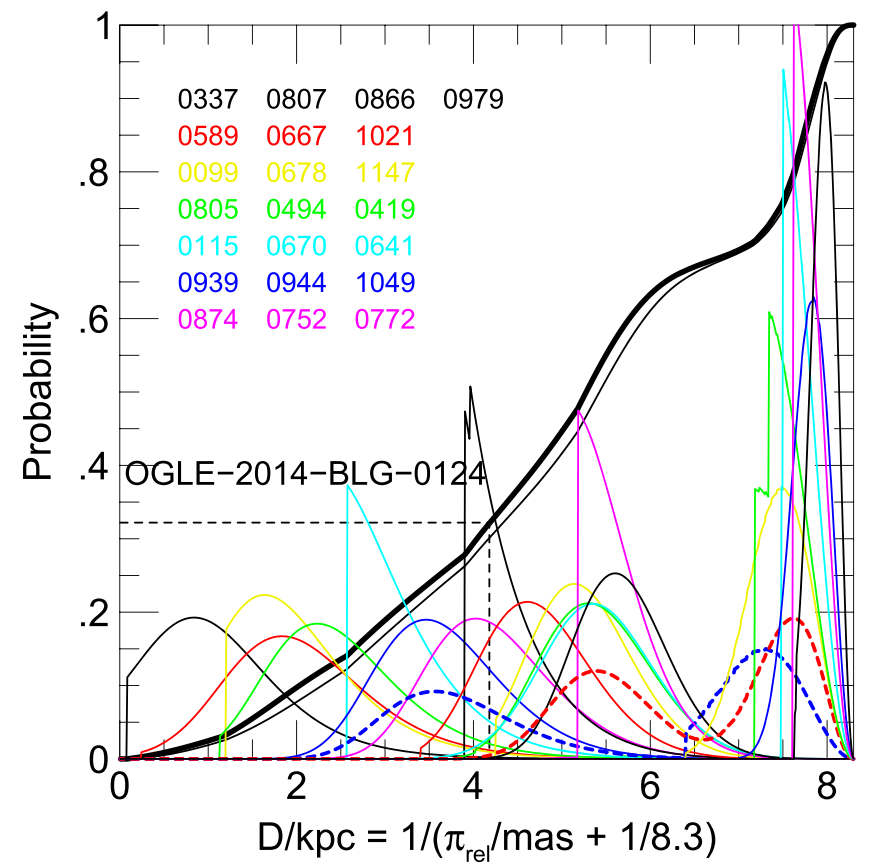

Figure 3. Distance estimates for each of the 21 lenses analyzed in the present paper plus OGLE-2014-BLG-0939 previously analyzed by Yee et al. (2015). The curves indicate the individual probability distributions. The corresponding event names are listed (upper left) in order of increasing mean estimated distance and are displayed in the same color as the distribution. The abscissa represents $D \equiv \mathrm{kpc} /\left(\pi_{\mathrm{rel}} / \mathrm{mas}+1 / 8.3\right)$ because it is the lens-source relative parallax $\pi_{\text {rel }}$ that is actually measured. With this display $D_{L} \sim D$ for $D \lesssim D_{S} / 2$ and $D_{S}-D_{L} \sim 8.3 \mathrm{kpc}-D$ for $D \gtrsim D_{S} / 2$. That is, the distance to the left boundary is very nearly the lens distance for the left half of the diagram and the distance to the right boundary is very nearly the distance between the lens and source for the right half. The value of $D$ for the one planet detected by Spitzer in this campaign (orbiting the lens star in the event OGLE-2014-BLG-0124; Udalski et al. 2015) is shown by a dashed line. By merging the results of several such campaigns, one would measure the Galactic distribution of planets between the solar circle and the Galactic bulge. The calculation assumes a prior that is flat in log-mass with hard cutoffs at $M<1.1 M_{\odot}$ (bulge) and $M<1.5 M_{\odot}$ (disk). The cumulative distribution is shown for this calculation (bold) and also for one with a realistic mass prior (solid). The difference is extremely small because the kinematic priors completely dominate. Two events shown in bold dashed curves (OGLE-2014-BLG-0944 and OGLE-2014-BLG1021 ) are the only ones with ambiguous (disk/bulge) distance determinations.

can be said about the Galactic distribution of planets based on a single planet. However, as emphasized in Section 1, events can be added from future observing campaigns by either Spitzer or other space observatories, with the isolated lenses forming the cumulative distribution function and the planetary events being used to measure the distance distribution of planets relative to this cumulative distribution.

Note that, in general, the individual distance measurements for the planetary events will be more accurate than for the isolated-lens events. This is because the former will mostly have measurements of $\theta_{\mathrm{E}}$ (from caustic crossings and/or approaches) and thus $\pi_{\text {rel }}=\theta_{\mathrm{E}} \pi_{\mathrm{E}}$, while the latter will have distance estimates based on measured $\tilde{\boldsymbol{v}}_{\text {hel }}$ combined with kinematic priors. However, because there are many more isolated-lens events than planetary events and because the kinematic distance estimates for the isolated lenses are relatively accurate (see Figure 3), uncertainties in the cumulative distribution function will not contribute much to uncertainties in the overall measurement. Rather, the precision of measurement of the Galactic distribution of planets will 
depend directly on how many planets are detected in spacebased parallax surveys.

There are essentially two ways to increase the number of planets detected in space-based campaigns. The first is simply to observe in additional years and/or with additional satellites. Both Spitzer and Kepler (in its K2 mode) are quite well suited to this task. The second is to make more intensive use of the time available for Galactic bulge observations. In the case of Kepler this is an automatic feature since Kepler observes its targets almost continuously as a matter of course. For Spitzer more intensive observing can increase the number of planetary detections in two ways: first by allowing more events to be monitored and second by detecting planets from space that are not detected from the ground. Because the spacecraft probes a region of the Einstein ring that is more or less separated from the one seen from the ground, it can observe planetary caustics that are not seen from the ground (Gould \& Horne 2013). However, this requires that the events be observed several to many times per day as compared to roughly once per day in the present campaign.

We note that roughly $30 \%$ of the lenses in our sample are in the bulge compared to roughly $60 \%$ expected for an unbiased sample of lensing events. Qualitatively, the reason for this is clear: the delay between recognition of the events and uploading coordinates to the spacecraft biases the sample to long events, which are preferentially in the disk. The same bias (for somewhat different reasons) affects the Gould et al. (2010) sample of high-magnification events.

This bias in the sample of underlying events does not in any way bias the measurement of the Galactic distribution of planets because the planetary events are subject to the same selection effects. However, to the extent that bulge events are underrepresented in the sample, it does mean that more planetary detections will be needed to measure the bulgeversus-disk fractions compared to what would be the case if there were more bulge events. Thus, it is important to develop more aggressive methods of identifying shorter events in time to upload coordinates, to the extent that this is possible.

Finally, we note the Galactic distribution of planets must be determined from the cumulative distribution (with distance) of planet sensitivity of events with parallaxes, not simply the cumulative distribution of the events themselves (as in Figure 3). Such planet-sensitivity calculations are an essential feature of all microlens planet frequency analyses. See, e.g., Figure 8 of Gaudi et al. (2002) or Figures 2-4 of Gould et al. (2010). Since microlens planet sensitivity is a function of both planet-star mass ratio $q$ and normalized separation $s$, such studies of the Galactic distribution of planets can in principle also yield functions of these variables. At the first stages, however, all that will be accessible is the distribution of a planet frequency that is suitably averaged over $q$ and $s$.

\section{CONCLUSIONS}

We have measured the microlens parallaxes of 21 events that were discovered by OGLE and observed by Spitzer, which was located $\sim 1$ AU west of Earth in projection. We used kinematic priors based on a Galactic model to estimate distances to each of the lenses. In the great majority of cases, these distributions are well localized, as illustrated in Figure 3. Such localization was not guaranteed in advance because the lens distances are subject to a well-known fourfold degeneracy (Refsdal 1966; Gould 1994).
In the case of 10 of the 21 events, we were able to break the key element of this degeneracy by quantifying and testing an argument originally given by J. Rich (circa 1997, private communication). In its quantified form, this states that, provided that $\pi_{\mathrm{E},-} \ll \pi_{\mathrm{E},+}$, the $\Delta u_{0,+, \pm}$ solutions (in which the source appears on the opposite side of the lens as seen from Earth and from the satellite) are less probable than the $\Delta u_{0,-, \pm}$ solutions by a factor $\sim\left(\pi_{\mathrm{E},-} / \pi_{\mathrm{E},+}\right)^{2} / 2$. The remaining degeneracy within the $\Delta u_{0,-, \pm}$ solutions is relatively unimportant because it leads to similar distance estimates and because, at least for disk lenses, the kinematic priors usually discriminate between these two solutions. As demonstrated by Figure 3, only two of the 21 events have substantially extended probability distributions of the distance variable $D \equiv \mathrm{kpc} /\left(\pi_{\mathrm{rel}} / \mathrm{mas}+1 / 8.3\right)$.

We have shown that an accurate cumulative distribution function of lens distances can be constructed from our sample. This means that the distances of planets detected from the same program can be used to determine the Galactic distribution of planets. That is, the Spitzer sample is a fair parent sample for the planets detected, even though the sample itself is biased toward longer events (and so disk lenses). The reason that this sample is nevertheless fair is that planetary events and the nonplanetary events suffer exactly the same bias because the planetary nature of the events is not known at the time the decision is made to observe them (e.g., Udalski et al. 2015). This means that this sample can be combined with future samples, including those observed in future years by Spitzer and Kepler, even though the selection biases of these samples are likely to differ radically.

Work by C.A.B. was carried out in part at the Jet Propulsion Laboratory (JPL), California Institute of Technology, under a contract with the National Aeronautics and Space Administration. Work by J.C.Y. A.G., and S.C. was supported by JPL grant 1500811. A.G. and B.S.G. were supported by NSF grant AST 1103471. A.G., B.S.G., and R. W.P. were supported by NASA grant NNX12AB99G. The OGLE project has received funding from the European Research Council under the European Community's Seventh Framework Programme (FP7/2007-2013)/ ERC grant agreement no. 246678 to A.U. Work by J.C.Y. was performed under contract with the California Institute of Technology (Caltech)/Jet Propulsion Laboratory (JPL) funded by NASA through the Sagan Fellowship Program executed by the NASA Exoplanet Science Institute. Work by D.D.P. and K.L. was supported by the University of Rijeka project 13.12.1.3.02. Work by T.S. is supported by grants JSPS23103002, JSPS24253004, and JSPS26247023. Work by I.A.B. was supported by the Marsden Fund of the Royal Society of New Zealand, contract no. MAU1104. The MOA project is supported by the grant JSPS25103508 and 23340064. Work by D.M. is supported by the I-CORE program of the Planning and Budgeting Committee and the Israel Science Foundation, Grant 1829/12. D.M. and A.G. acknowledge support by the US-Israel Binational Science Foundation. The operation of the Danish $1.54 \mathrm{~m}$ telescope at ESO's La Silla Observatory is financed by a grant to U.G.J. from the Danish Natural Science Foundation (FNU). This publication was made possible by NPRP grants 09-476-1-078 and X-019-1-006 from the Qatar National Research Fund (a member of Qatar Foundation). O.W. (FNRS research fellow) 
and J. Surdej acknowledge support from the Communauté française de Belgique-Actions de recherche concertéesAcadémie Wallonie-Europe. M.H. acknowledges support from the Villum Foundation. C.S. received funding from the European Union Seventh Framework Programme (FP7/ 2007-2013) under grant agreement no. 268421. This work is based in part on observations made with the Spitzer Space Telescope, which is operated by the Jet Propulsion Laboratory, California Institute of Technology under a contract with NASA. The research described in this publication was carried out in part at the Jet Propulsion Laboratory, California Institute of Technology, under a contract with the National Aeronautics and Space Administration.

\section{REFERENCES}

Alard, C., \& Lupton, R. H. 1998, ApJ, 503, 325

Albrow, M., Beaulieu, J.-P., Birch, P., et al. 1998, ApJ, 509, 687

Bond, I. A., Abe, F., Dodd, R. J., et al. 2001, MNRAS, 327, 868

DePoy, D. L., Atwood, B., Belville, S. R., et al. 2003, SPIE, 4841, 827

Dominik, M., Jørgensen, U. G., Rattenbury, N. J., et al. 2010, AN, 331,671

Dong, S., Udalski, A., Gould, A., et al. 2007, ApJ, 664, 862

Fazio, G. G., Hora, J. L., Allen, L. E., et al. 2004, ApJS, 154, 10

Gaudi, B. S. 2012, ARA\&A, 50, 411

Gaudi, B. S., Albrow, M. D., \& An, J. 2002, ApJ, 566, 463

Gould, A. 1994, ApJL, 421, L71

Gould, A. 1997, ApJ, 480, 188
Gould, A. 1999, ApJ, 514, 869

Gould, A. 2000, ApJ, 542, 785

Gould, A. 2004, ApJ, 606, 319

Gould, A., Dong, S., Gaudi, B. S., et al. 2010, ApJ, 720, 1073

Gould, A., \& Horne, K. 2013, ApJL, 779, L28

Gould, A., Udalski, A., Monard, B., et al. 2009, ApJL, 698, L147

Gould, A., \& Yee, J. C. 2013, ApJ, 764, 107

Han, C., \& Gould, A. 1995, ApJ, 447, 53

Henderson, C. B., Gaudi, B. S., Han, C., et al. 2014, ApJ, 794, 52

Jiang, G., DePoy, D. L., Gal-Yan, A., et al. 2005, ApJ, 617, 1307

Liebes, S. 1964, PhRv, 133, 835

Makovoz, D., \& Marleau, F. R. 2005, PASP, 117, 1113

Muraki, Y., Han, C., Bennett, D. P., et al. 2011, ApJ, 741, 22

Paczyński, B. 1986, ApJ, 304, 1

Poindexter, S., Afonso, C., Bennett, D. P., et al. 2005, ApJ, 633, 914

Refsdal, S. 1964, MNRAS, 128, 295

Refsdal, S. 1966, MNRAS, 134, 315

Schechter, P. L., Mateo, M., \& Saha, A. 1993, PASP, 105, 1342

Shvartzvald, Y., \& Maoz, D. 2012, MNRAS, 419, 3631

Simpson, T. 1740, Essays on Several Curious and Useful Subjects: In Speculative and Mixd Mathematicks (London: H. Woodfall Jr.)

Skowron, J., Udalski, A., Gould, A., et al. 2011, ApJ, 738, 87

Sumi, T., Bennett, D. P., Bond, I. A., et al. 2013, ApJ, 778, 150

Tsapras, Y., Street, R., Horne, K., et al. 2009, AN, 330, 4

Udalski, A. 2003, AcA, 53, 291

Udalski, A., Szymanski, M., Kaluzny, J., et al. 1994, AcA, 44, 227

Udalski, A., Yee, J. C., Gould, A., et al. 2015, ApJ, 799, 237

Werner, M. W., Roellig, T. L., Low, F. J., et al. 2004, ApJS, 154, 1

Yee, J. C., Shvartzvald, Y., Gal-Yam, A., et al. 2012, ApJ, 755, 102

Yee, J. C., Udalski, A., Calchi Novati, S., et al. 2015, ApJ, 802, 76

Yee, J. C., Udalski, A., Sumi, T., et al. 2009, ApJ, 703, 2082 